\title{
Chromaticity effects on head-tail instabilities for broadband impedance using two particle model, Vlasov analysis, and simulations
}

\author{
Yong Ho Chin \\ High Energy Accelerator Research Organization (KEK), 1-1 Oho, Tsukuba, Ibaraki-ken 305-0801, Japan
}

Alexander Wu Chao

SLAC National Accelerator Laboratory, 2575 Sand Hill Road, Menlo Park, California 94025, USA

Michael M. Blaskiewicz

Brookhaven National Laboratory (BNL), P.O. Box 5000, Upton, New York 11973-5000, USA

Yoshihiro Shobuda

Japan Atomic Energy Agency (JAEA), 2-4 Shirakata, Tokaimura, Nakagun, Ibaraki 319-1195, Japan

(Received 31 August 2016; revised manuscript received 26 June 2017; published 28 July 2017)

\begin{abstract}
Effects of the chromaticity on head-tail instabilities for broadband impedances are comprehensively studied, using the two particle model, the Vlasov analysis and computer simulations. We show both in the two particle model and the Vlasov analysis with the trapezoidal (semiconstant) wake model that we can derive universal contour plots for the growth factor as a function of the two dimensionless parameters: the wakefield strength, $\Upsilon$, and the difference of the betatron phase advances between the head and the tail, $\chi$. They reveal how the chromaticity affects strong head-tail instabilities and excites head-tail instabilities. We also apply the LEP (Large Electron-Positron Collider) broadband resonator model to the Vlasov approach and find that the results are in very good agreement with those of the trapezoidal wake model. The theoretical findings are also reinforced by the simulation results. The trapezoidal wake model turns out to be a very useful tool since it significantly simplifies the time domain analysis and provides well-behaved impedance at the same time.
\end{abstract}

DOI: 10.1103/PhysRevAccelBeams.20.071003

\section{INTRODUCTION}

Effects of the chromaticity on head-tail modes are one of classical subjects in the beam instability theory and have been extensively studied both theoretically and by simulations. One approach is to start with the Vlasov equation and to solve it using the mode expansion technique (both azimuthally and radially) [1]. However, when it is applied to a broadband impedance, many azimuthal and radial modes are mixed up in a complex way, and computational results often do not converge smoothly, in particular with large chromaticities, as a function of number of azimuthal and radial modes included in the calculations.

The two particle model has been applied to illustrate the mechanism of the strong head-tail instability [or the transverse mode-coupling instability (TMCI)] in a very simple but insightful way (Ref. [2], page 179, and Kohaupt [3]). It is a quite effective tool to get a simple picture of the essence of complicated physical phenomena. It has been

\footnotetext{
*yongho.chin@kek.jp

Published by the American Physical Society under the terms of the Creative Commons Attribution 3.0 License. Further distribution of this work must maintain attribution to the author(s) and the published article's title, journal citation, and DOI.
}

also expanded to include chromaticity effects in Chao's book (page 197). The chromaticity introduces the energyerror dependence to the betatron frequencies. This small additional term, however, makes it very difficult to find exact solutions even for the free betatron oscillation. Only the perturbation solution for a small chromaticity and weak wakefield strength is presented in Chao's book. [Talman [4] and Lee and Peggs [5] use the two particle model for similar problems, but they assume either a small chromaticity or use a different impedance model (resistive wall) from the present study. They are very good references to understand the history of development of TMCI theory.]

In this paper, we present two approaches to solve the two particle model with (any value of) chromaticity. We consider only short range wakefield effects (no wake left from previous turns) and neglect space charge effects. An ultrarelativistic beam is assumed. The final results are summarized as universal contour plots for three dimensionless parameters. They demonstrate how the chromaticity affects strong head-tail instabilities and excites head-tail instabilities in a clear fashion.

We then move to the Vlasov analysis with a Gaussian bunch distribution to compare its results with those of the two particle model. For this end, we introduce the trapezoidal model to solve the singularity problem of the naive 
constant wake model. We also employ the LEP (Large Electron-Positron Collider) broadband resonator model to see how the impedance model will change the behavior of the head-tail instabilities. Simulations are carried out to see how they reinforce the theoretical findings.

We briefly summarize Chao's two particle model with the chromaticity in Sec. II. In Sec. III, we introduce Hill's equation technique to show how the equations of motion with the chromaticity can be solved conceptually without the assumption of small chromaticity. The explicit solutions are derived in Sec. IV. The formula for the growth rate, and its three-dimensional and flat contour plots are presented in Sec. V. We then show another approach using the square well model in Sec. VI to solve the two particle model. The two particle mode is concluded with its findings in Sec. VII. We then move to the Vlasov analysis to the present problem in Sec. VIII for more accurate predictions, and compare Vlasov results with those of the two particle model. We also apply the simulation approach in Sec. IX to illustrate that our findings with the Vlasov analysis are consistent with simulations. The paper is concluded in Sec. X.

\section{TWO PARTICLE MODEL WITH CHROMATICITY}

Let us first review Chao's two particle model with the chromaticity by closely following his textbook. We assume that a beam is made of two macroparticles, each with charge of $\mathrm{Ne} / 2$ and each executing smooth synchrotron and betatron oscillations. We assume that their synchrotron oscillations have equal amplitude, but opposite phases. As for the betatron oscillations, we make no such assumption for the amplitude and the phase.

In what follows, we use $s$, the distance along the circumference, as an independent variable of motion. During the first half of the synchrotron oscillation period, $\mathrm{T}_{s}=2 \pi / \omega_{s}$, particle 1 leads particle 2 on the synchrotron phase space, and only the trailing particle (particle 2 in the present case) receives transverse kicks from wakefields created by the leading particle (particle 1 in the present case), which is a function of the transverse displacement of the leading particle. For simplicity, we assume that the wake potential is a constant $W_{0}$, independent of the distance between the two particles. The property of the wake potential requires that $W_{0}>0$.

We assume that the synchrotron oscillations of the two particles are given by

$$
z_{1}=\hat{z} \sin \frac{\omega_{s} s}{c},
$$

and

$$
z_{2}=-z_{1},
$$

where $\omega_{s}$ is the synchrotron oscillation frequency and $c$ is the speed of light. The chromaticity introduces the energy- error $(\delta=\Delta E / E)$ dependence to the betatron oscillation frequencies as

$$
\omega_{\beta}(\delta)=\omega_{\beta}(1+\xi \delta),
$$

where $\omega_{\beta}$ is the betatron oscillation frequency for an on-momentum particle and $\xi$ is the chromaticity. During the first half of the synchrotron oscillation period, $0<\mathrm{s} / \mathrm{c}<T_{s} / 2$, or $0<\mathrm{s}<\pi c / \omega_{s}$, the betatron oscillation frequencies can be written more specifically as

$$
\omega_{\beta}\left(\delta_{1}\right)=\omega_{\beta}\left(1-\frac{\xi \omega_{s} \hat{z}}{c \eta} \cos \frac{\omega_{s} s}{c}\right)
$$

and

$$
\omega_{\beta}\left(\delta_{2}\right)=\omega_{\beta}\left(1+\frac{\xi \omega_{s} \hat{z}}{c \eta} \cos \frac{\omega_{s} s}{c}\right)
$$

where $\eta$ is the slippage factor.

The equations of motion for the two particles are written as

$$
y_{1}^{\prime \prime}+\left[\frac{\omega_{\beta}\left(\delta_{1}\right)}{c}\right]^{2} y_{1}=0
$$

and

$$
y_{2}^{\prime \prime}+\left[\frac{\omega_{\beta}\left(\delta_{2}\right)}{c}\right]^{2} y_{2}=\frac{N r_{0} W_{0}}{2 \gamma C} y_{1},
$$

where $y^{\prime}=\frac{d y}{d s}, \gamma$ is the Lorenz factor, $C$ is the circumference of the machine, and $r_{0}$ is the classical radius of the particle. Similarly, during the second half period of the synchrotron oscillation, we have the same equations with indices 1 and 2 exchanged.

\section{HILL's EQUATION}

Let us solve the homogenous equation (6) first. This equation is a Hill's equation [6] with a periodic function of $K(\mathrm{~s})$ whose period is $T_{s} c$, namely, $K\left(s+T_{s} c\right)=K(s)$ :

$$
y^{\prime \prime}+K(s) y=0 .
$$

A general solution of Hill' Eq. (8) is

$$
y=w(s) e^{i \Psi(s)},
$$

where the functions $w(s)$ and $\Psi(s)$ satisfy the following equations:

$$
w^{\prime \prime}-\frac{1}{w^{3}}+K w=0,
$$

and 


$$
\Psi^{\prime}=\frac{1}{w^{2}}
$$

Let us define "Twiss parameters" $\beta$ and $\alpha$ as

$$
\beta=w^{2},
$$

and

$$
\alpha=-w w^{\prime}=-\frac{\beta^{\prime}}{2} .
$$

From Eq. (11), the phase advance $\Psi(s)$ can be written using $\beta$ as

$$
\Psi=\int_{0}^{s} \frac{d s}{\beta} .
$$

Then, the general solution (9) can be expressed as

$$
y(s)=a \sqrt{\beta} \cos \int_{0}^{s} \frac{d s}{\beta},
$$

where the coefficient $a$ is a constant of motion.

The solution of equation of motion (6) is thus given by

$$
y_{1}(s)=a_{1} \sqrt{\beta_{1}(s)} \cos \int_{0}^{s} \frac{d s}{\beta_{1}(s)},
$$

where the function $\beta_{1}$ satisfies Eqs. (10) and (12) when the function $K(s)$ is equated to

$$
K_{1}(s)=\left[\frac{\omega_{\beta}\left(\delta_{1}\right)}{c}\right]^{2} .
$$

If we define a new coordinate $\hat{y}_{1}$ for particle 1 as

$$
\hat{y}_{1}(s)=y_{1}+i\left(\beta_{1} y_{1}^{\prime}+\alpha_{1} y_{1}\right),
$$

its motion is given by

$$
\hat{y}_{1}(s)=a_{1} \sqrt{\beta_{1}(s)} \exp \left[-i \int_{0}^{s} \frac{d s}{\beta_{1}(s)}\right] .
$$

\section{SOLUTION FOR PARTICLE 2}

Let us solve the equation of motion (8) for particle 2 . We define a new coordinate $\hat{y}_{2}$ for particle 2 as

$$
\hat{y}_{2}(s)=y_{2}+i\left(\beta_{2} y_{2}^{\prime}+\alpha_{2} y_{2}\right) \text {, }
$$

where the functions $\beta_{2}$ and $\alpha_{2}$ satisfy Eqs. (10), (12) and (13) when the function $K(s)$ is equated to

$$
K_{2}(s)=\left[\frac{\omega_{\beta}\left(\delta_{2}\right)}{c}\right]^{2}
$$

By taking the derivative of $\hat{y}_{2}$ and using Eq. (7), we obtain an equation of motion for particle 2 (see Appendix A):

$$
\hat{y}_{2}^{\prime}=\frac{1}{i \beta_{2}} \hat{y}_{2}\left(1-i \alpha_{2}\right)+i \beta_{2} W y_{1},
$$

where we define $W$ as

$$
W=\frac{N r_{0} W_{0}}{2 \gamma C}
$$

Let us assume that the solution of the equation of motion for particle 2 has a form

$$
\hat{y}_{2}(s)=a_{2}(s) \sqrt{\beta_{2}(s)} \exp \left[-i \int_{0}^{s} \frac{d s}{\beta_{2}(s)}\right] \text {, }
$$

where the coefficient $a_{2}(s)$ is a function of $s$ and may not be a constant of motion. By taking the derivative of both sides of Eq. (24) and equating them with Eq. (22) (see Appendix A), we get

$$
a_{2}^{\prime}(s) \sqrt{\beta_{2}} \exp \left[-i \int_{0}^{s} \frac{d s}{\beta_{2}}\right]=i \beta_{2} W y_{1}
$$

The solution of the above equation is (see Appendix A)

$$
\begin{aligned}
a_{2}(s)= & a_{2}(0)+i \frac{W}{2} a_{1}(0) \int_{0}^{s} \sqrt{\beta_{1} \beta_{2}}\left[\operatorname { e x p } \left(i \int_{0}^{s} \frac{d s}{\beta_{1}}\right.\right. \\
& \left.\left.+i \int_{0}^{s} \frac{d s}{\beta_{2}}\right)+\exp \left(-i \int_{0}^{s} \frac{d s}{\beta_{1}}+i \int_{0}^{s} \frac{d s}{\beta_{2}}\right)\right] d s .
\end{aligned}
$$

So far, no approximation has been made. To proceed further, we need to know a concrete form of the function $\beta$. The function $\beta\left(=w^{2}\right)$ is a solution of Eq. (10). If we neglect the $w^{\prime \prime}$ term in Eq. (10) assuming that $w$ changes slowly in time, an approximate solution of $w$ is given by

$$
\frac{1}{w^{2}}=\sqrt{K}=\frac{1}{\beta} .
$$

Therefore, the inverse of the function $\beta$ is the betatron frequency itself: 


$$
\begin{aligned}
\frac{1}{\beta_{1}} & =\frac{\omega_{\beta}}{c}\left(\delta_{1}\right) \\
& =\frac{\omega_{\beta}}{c}\left(1-\frac{\xi \omega_{s} \hat{z}}{c \eta} \cos \frac{\omega_{s} s}{c}\right) \stackrel{\text { def }}{=} \frac{\omega_{\beta}}{c}\left(1-\varepsilon \cos \frac{\omega_{s} s}{c}\right),
\end{aligned}
$$

and

$$
\begin{aligned}
\frac{1}{\beta_{2}} & =\frac{\omega_{\beta}}{c}\left(\delta_{2}\right) \\
& =\frac{\omega_{\beta}}{c}\left(1+\frac{\xi \omega_{s} \hat{z}}{c \eta} \cos \frac{\omega_{s} s}{c}\right) \stackrel{\operatorname{def}}{=} \frac{\omega_{\beta}}{c}\left(1+\varepsilon \cos \frac{\omega_{s} s}{c}\right) .
\end{aligned}
$$

Here, we define the parameter $\varepsilon$ as

$$
\varepsilon=\frac{\xi \omega_{s} \hat{z}}{c \eta} .
$$

The difference of the betatron phase advances between the head and the tail is given by the parameter [2]

$$
\chi=2 \frac{\xi \omega_{\beta} \hat{z}}{c \eta} .
$$

It is typically on the order of $1-10$. The parameter $\varepsilon$ is related to $\chi$ as

$$
\varepsilon=\chi \cdot \frac{1}{2} \frac{\omega_{s}}{\omega_{\beta}} .
$$

Since the synchrotron oscillation is much slower than the betatron oscillation $\left(\frac{\omega_{s}}{\omega_{\beta}} \ll 1\right)$, the parameter $\varepsilon$ is much smaller than 1:

$$
\varepsilon \ll 1
$$

Therefore, the factor $\sqrt{\beta_{1} \beta_{2}}$ in Eq. (26) can be approximated as

$$
\sqrt{\beta_{1} \beta_{2}}=\frac{c}{\omega_{\beta} \sqrt{1-\left(\varepsilon \cos \frac{\omega_{s} s}{c}\right)^{2}}} \cong \frac{c}{\omega_{\beta}} .
$$

By inserting Eqs. (28) and (29) to Eq. (26) and using Eq. (34), we finally obtain

$$
\begin{aligned}
a_{2}(s) \cong & a_{2}(0)+i \frac{W}{2} a_{1}(0) \frac{c}{\omega_{\beta}} \int_{0}^{s}\left[\exp \left(2 i \frac{\omega_{\beta}}{c} s\right)\right. \\
& \left.+\exp \left(i \chi \sin \frac{\omega_{s}}{c} s\right)\right] d s .
\end{aligned}
$$

The first term in the bracket can be dropped because it is much smaller than the second term by a factor of $\frac{\omega_{\beta}}{\omega_{s}}$. The second term is the resonant response with the chromatic effect.
After some tedious but straightforward calculations (see Appendix B), Eq. (35) at the end of the first half of the synchrotron oscillation period $\left(s=\frac{T_{s} c}{2}=\frac{\pi c}{\omega_{s}}\right)$ can be simplified as

$$
a_{2}\left(\frac{\pi c}{\omega_{s}}\right)=a_{2}(0)+i a_{1}(0) \cdot \Upsilon \cdot f(\chi)
$$

where we define a positive dimensionless parameter $\Upsilon$ for the wakefield strength

$$
\mathrm{r}=\frac{\pi N r_{0} W_{0} c^{2}}{4 \gamma C \omega_{\beta} \omega_{s}}
$$

Here, the function $f(\chi)$ is a complex function

$$
f(\chi)=h(\chi)+\mathrm{i} g(\chi),
$$

where

$$
\begin{gathered}
h(\chi)=J_{0}(\chi), \\
g(\chi)=\sum_{m=0}^{\infty} J_{(2 m+1)}(\chi) \frac{4}{(2 m+1) \pi}
\end{gathered}
$$

and $J_{n}(\chi)$ is the Bessel function of the first kind. The transformation from $\mathrm{s}=0$ to $s=\frac{T_{s} c}{2}=\frac{\pi c}{\omega_{s}}$ is thus described in a matrix form as

$$
\left[\begin{array}{l}
a_{1} \\
a_{2}
\end{array}\right]_{s=\pi c / \omega_{s}}=\left[\begin{array}{cc}
1 & 0 \\
i \Gamma & 1
\end{array}\right]\left[\begin{array}{l}
a_{1} \\
a_{2}
\end{array}\right]_{s=0},
$$

where we have defined a dimensionless parameter $\Gamma$ as

$$
\Gamma=\Upsilon \cdot f(\chi) .
$$

Figure 1 shows the function $f(\chi)$. The real and the imaginary parts of $f(\chi)$, namely, $h(\chi)$ and $g(\chi)$, are denoted by the blue solid and the blue dashed lines, respectively.

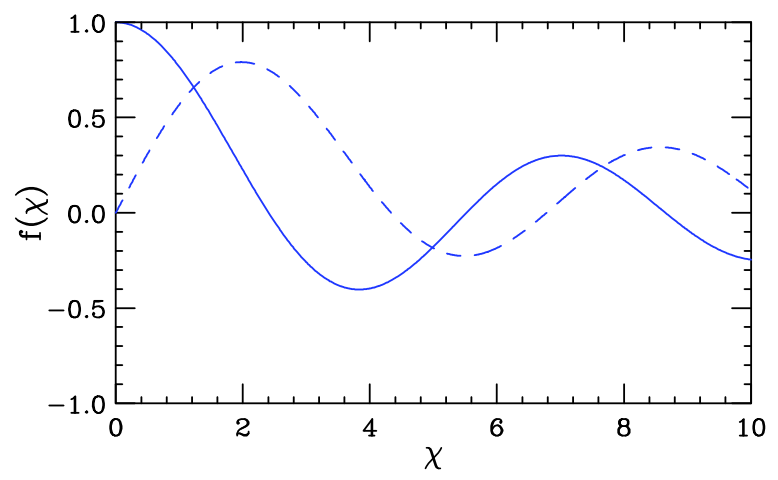

FIG. 1. The complex function $f(\chi)$ as a function of $\chi$. The real and the imaginary parts of $f(\chi)$, namely, $h(\chi)$ and $g(\chi)$, are denoted by the blue solid and the blue dashed lines, respectively. 
The first root of $h(\chi)$ is $\chi=2.41$. The second and the third roots of $g(\chi)$ are $\chi=4.33$ and $\chi=6.78$, respectively.

It is interesting to point out that the parameter $\Gamma$ is identical to Chao's $\Upsilon$ in Eq. (4.100) (on page 202 in Ref. [2]), though the notations are slightly different. Equation (4.100) turns out to be the exact solution for any $\chi$, not limited to a small value.

\section{EIGENVALUES}

The transfer matrix during the second half of the synchrotron oscillation period, $T_{s} / 2<s / c<T_{s}$, or $\pi c / \omega_{s}<s<2 \pi c / \omega_{s}$, is obtained by exchanging the indices 1 and 2 in Sec. III. It is then

$$
\left[\begin{array}{l}
a_{1} \\
a_{2}
\end{array}\right]_{s=2 \pi c / \omega_{s}}=\left[\begin{array}{cc}
1 & i \Gamma \\
0 & 1
\end{array}\right]\left[\begin{array}{l}
a_{1} \\
a_{2}
\end{array}\right]_{s=\pi c / \omega_{s}} .
$$

The total transfer matrix for one full synchrotron oscillation period is then given by

$$
\begin{aligned}
{\left[\begin{array}{l}
a_{1} \\
a_{2}
\end{array}\right]_{s=2 \pi c / \omega_{s}} } & =\left[\begin{array}{ll}
1 & i \Gamma \\
0 & 1
\end{array}\right]\left[\begin{array}{cc}
1 & 0 \\
i \Gamma & 1
\end{array}\right]\left[\begin{array}{l}
a_{1} \\
a_{2}
\end{array}\right]_{s=0} \\
& =\left[\begin{array}{cc}
1-\Gamma^{2} & i \Gamma \\
i \Gamma & 1
\end{array}\right]\left[\begin{array}{l}
a_{1} \\
a_{2}
\end{array}\right]_{s=0} .
\end{aligned}
$$

Let us find eigenvalues $\lambda$ of this matrix by equating it as

$$
\left[\begin{array}{cc}
1-\Gamma^{2} & i \Gamma \\
i \Gamma & 1
\end{array}\right]\left[\begin{array}{l}
a_{1} \\
a_{2}
\end{array}\right]=\lambda\left[\begin{array}{ll}
1 & 0 \\
0 & 1
\end{array}\right]\left[\begin{array}{l}
a_{1} \\
a_{2}
\end{array}\right]
$$

The eigenvalues are found to be

$$
\lambda=1-\frac{\Gamma^{2}}{2} \pm \sqrt{\frac{\Gamma^{2}}{2} \cdot\left(\frac{\Gamma^{2}}{2}-2\right)} .
$$

They can be more explicitly expressed as

$$
\lambda= \begin{cases}(1-H)-i G \pm \frac{1}{\sqrt{2}}\left(\sqrt{a+\sqrt{a^{2}+b^{2}}}+i \sqrt{-a+\sqrt{a^{2}+b^{2}}}\right) & \text { if } b \geq 0 \\ (1-H)-i G \pm \frac{1}{\sqrt{2}}\left(\sqrt{a+\sqrt{a^{2}+b^{2}}}-i \sqrt{-a+\sqrt{a^{2}+b^{2}}}\right) & \text { if } b \leq 0\end{cases}
$$

where

$$
\begin{gathered}
H(\chi)=\frac{\Upsilon^{2}}{2}\left[h^{2}(\chi)-g^{2}(\chi)\right], \\
G(\chi)=\frac{\Upsilon^{2}}{2} 2 h(\chi) g(\chi),
\end{gathered}
$$

and

$$
a \stackrel{\text { def }}{=} H^{2}-G^{2}-2 H
$$

$$
b \stackrel{\text { def }}{=} 2 G(H-1) .
$$

The absolute value of $\lambda$ is given by

$$
|\lambda|= \begin{cases}\sqrt{\left((1-H) \pm \frac{1}{\sqrt{2}} \sqrt{a+\sqrt{a^{2}+b^{2}}}\right)^{2}+\left(-G \pm \frac{1}{\sqrt{2}} \sqrt{-a+\sqrt{a^{2}+b^{2}}}\right)^{2}} & \text { if } b \geq 0 \\ \sqrt{\left((1-H) \pm \frac{1}{\sqrt{2}} \sqrt{a+\sqrt{a^{2}+b^{2}}}\right)^{2}+\left(-G \mp \frac{1}{\sqrt{2}} \sqrt{-a+\sqrt{a^{2}+b^{2}}}\right)^{2}} & \text { if } b \leq 0 .\end{cases}
$$

The growth rate $g$ is given by

$$
e^{g T_{s}}=|\lambda|
$$

The "growth factor" $g \times T_{s}$ over one synchrotron oscillation period is given by

$$
g \times T_{s}=\log |\lambda|
$$

for given dimensionless parameters $\Upsilon$ and $\chi$.

We calculate the growth factor $g \times T_{s}$ as a function of $\Upsilon$ and $\chi$ for positive $\chi$ and plot it in a three-dimensional contour plot in Fig. 2 and in a flat contour plot in Fig. 3. The three parameters $g \times T_{s}, \Upsilon$ and $\chi$ are all dimensionless and these contour plots are universal. 


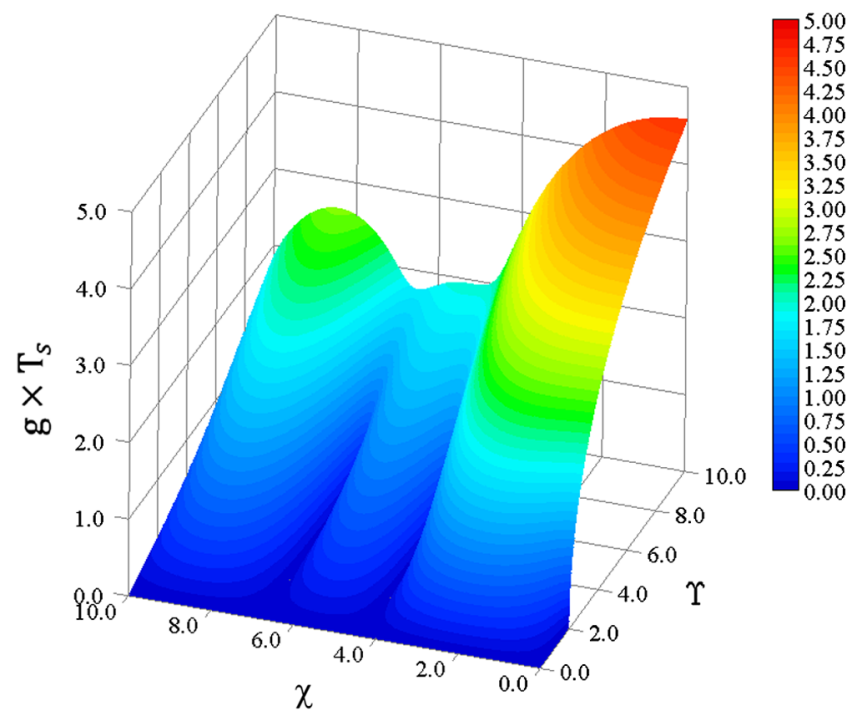

FIG. 2. Three-dimensional contour plot for the growth factor $g \times T_{s}$ as a function of $\Upsilon$ and $\chi$ for positive $\chi$.

Figure 4 shows a flat contour plot of the growth factor $g \times T_{s}$ as a function of $\Upsilon$ and $\chi$ for negative $\chi$. It can be seen that the growth factor is symmetrical for positive and negative $\chi$.

\section{SQUARE WELL MODEL}

There is another simple way to solve Eqs. (7) and (8). Let us approximate the parabolic rf potential by a square well one. In this case, a particle moves along the square orbit in the longitudinal phase space instead of the circle (it is just drifting between the two stone walls and bounces back at the walls) as shown in Fig. 5.

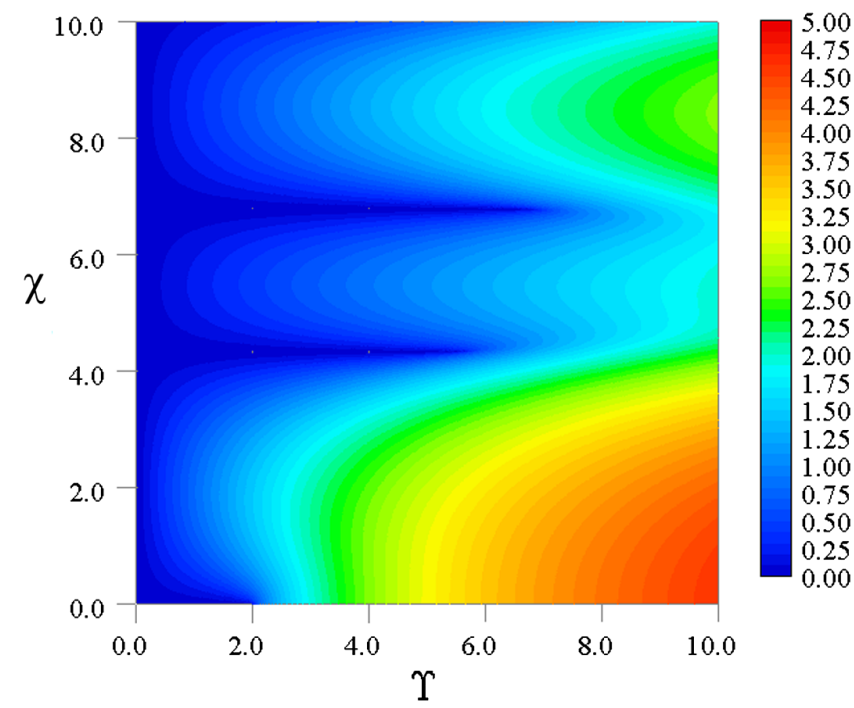

FIG. 3. Flat contour plot for the growth factor $g \times T_{s}$ as a function of $\Upsilon$ and $\chi$ for positive $\chi$.

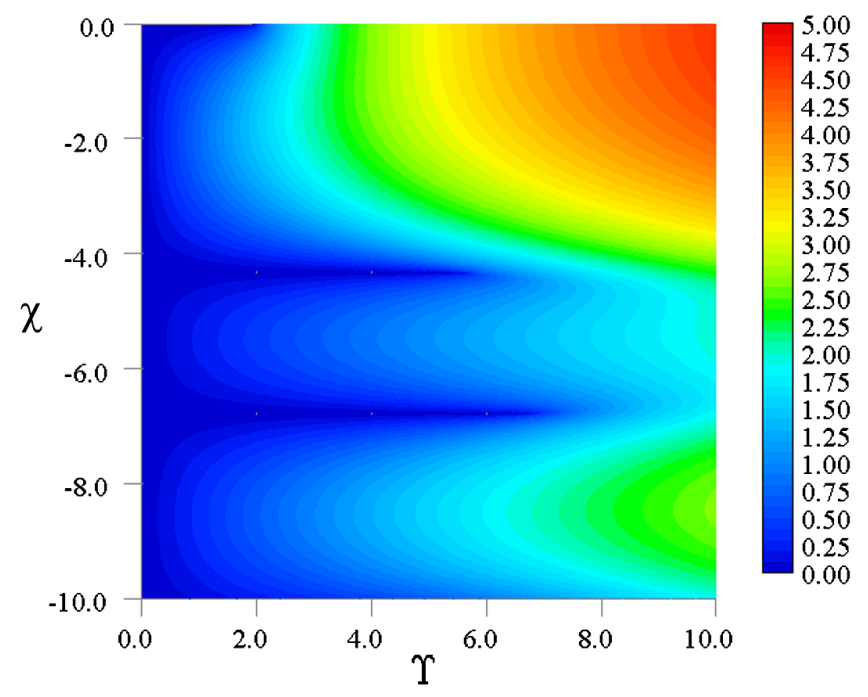

FIG. 4. Flat contour plot for the growth factor $g \times T_{s}$ as a function of $\Upsilon$ and $\chi$ for negative $\chi$.

Then, Eqs. (7) and (8) are approximated during the first half of the synchrotron oscillation period $0<s / c<T_{s} / 2$, or $0<s<\pi c / \omega_{s}$ as

$$
y_{1}^{\prime \prime}+\left(\frac{\omega_{\beta}}{c}\right)^{2}\left[1-\varepsilon_{s} c(s)\right]^{2} y_{1}=0,
$$

and

$$
y_{2}^{\prime \prime}+\left(\frac{\omega_{\beta}}{c}\right)^{2}\left[1+\varepsilon_{s} c(s)\right]^{2} y_{2}=\frac{N r_{0} W_{0}}{2 \gamma C} y_{1},
$$

where

$$
\varepsilon_{s}=\frac{\xi \omega_{s} \hat{z}_{s}}{c \eta}
$$

and

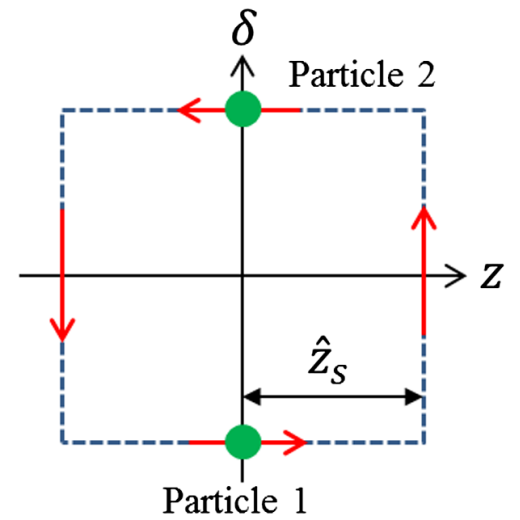

FIG. 5. The particle motion in the square well potential above the transition. 


$$
c(s)= \begin{cases}1, & 0<0<s<\pi c / 2 \omega_{s} \\ -1, & \pi c / 2 \omega_{s}<0<s<\pi c / \omega_{s}\end{cases}
$$

Remember that $s=\pi c / 2 \omega_{s}$ is a quarter of the synchrotron oscillation period. Here, $\hat{z}_{s}$ is a half distance between the two stone walls (the bunch length). It has to be determined so that the square well potential will accurately approximate the corresponding parabolic potential. The most important physical effect of the chromaticity is to produce the difference of the betatron phases between the two particles. This phase difference is always changing in both models since the two particles are moving along their orbits. Thus, for the two models to be comparable, the average value of the difference of the betatron phases between the two particles has to be equal in the two models. Then, we find (see Appendix C)

$$
\hat{z}_{s}=\frac{8}{\pi^{2}} \hat{z}
$$

Namely, the bunch length in the square well potential should be shorter than that in the parabolic potential by a factor of $\pi^{2} / 8$.

We assume

$$
y_{m}(s)=\hat{y}_{m}(s) \exp \left(-i \omega_{\beta} \frac{s}{c}\right), \quad m=1 \quad \text { or } 2,
$$

and look for a transfer matrix for $\hat{y}_{m}(s)$ from $s=0$ to $s=\pi c / \omega_{s}$. After some mathematical manipulations (see Appendix C), we get

$$
\left[\begin{array}{l}
\hat{y}_{1} \\
\hat{y}_{2}
\end{array}\right]_{s=\pi c / \omega_{s}}=\left[\begin{array}{cc}
1 & 0 \\
i \Gamma & 1
\end{array}\right]\left[\begin{array}{l}
\hat{y}_{1} \\
\hat{y}_{2}
\end{array}\right]_{s=0}
$$

where

$$
\Gamma=\Upsilon \cdot f_{s}(\chi)
$$

and

$$
f_{s}(\chi)=\exp \left(i \frac{2 \chi}{\pi}\right) \cdot \frac{\sin \frac{2 \chi}{\pi}}{\frac{2 \chi}{\pi}} .
$$

Figure 6 plots the complex function $f_{s}(\chi)$. The real and the imaginary parts of $f_{s}(\chi)$ are denoted by the green solid and the green dashed lines, respectively. For comparison, the function $f(\chi)$ for the parabolic potential is also plotted. Its real and imaginary parts are denoted by the blue solid and the blue dashed lines, respectively. The function $f_{s}(\chi)$ agrees very well with $f(\chi)$ up to $\chi \approx 1.5$. The imaginary part of $f_{s}(\chi)$ never goes to negative, while that of $f(\chi)$ oscillates between positive and negative signs.

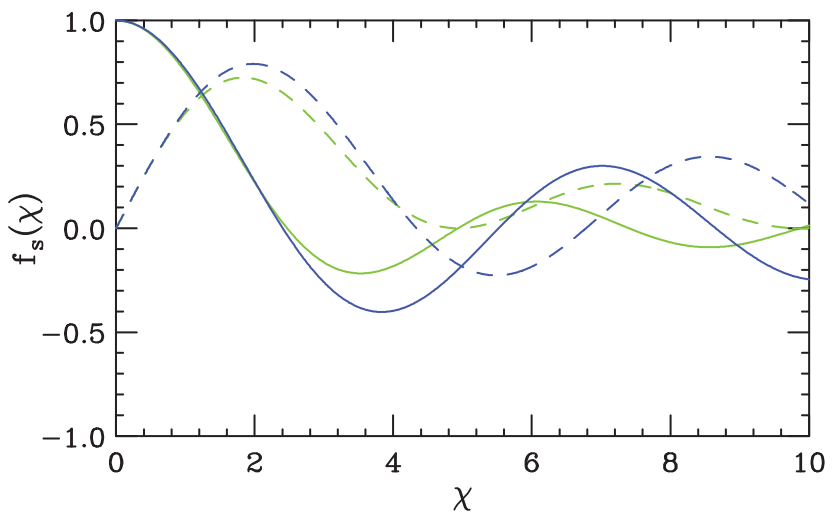

FIG. 6. The complex function $f_{s}(\chi)$. The real and the imaginary parts of $f_{s}(\chi)$ are denoted by the green solid and the green dashed lines, respectively. For comparison, the function $f(\chi)$ for the parabolic potential is also plotted. Its real and imaginary parts are denoted by the blue solid and the blue dashed lines, respectively.

Since the transfer matrix in Eq. (61) has the same form as that of the parabolic potential [the matrix in Eq. (41)], the arguments in Sec. IV can be applied here exactly as they are. The three-dimensional and the flat contour plots of the growth factor $g \times T_{s}$ are shown in Figs. 7 and 8, respectively, as a function of $\Upsilon$ and $\chi$ for positive $\chi$. They are similar to those for the parabolic potential up to $\Upsilon \approx 4$. The contour plots for the square well model show wide stable regions at around $\chi=5$ and $\chi=10$. The growth factor behaves symmetrically for positive and negative $\chi$, expected from the $\chi$ dependence in Eq. (62) similar to that of the parabolic potential case.

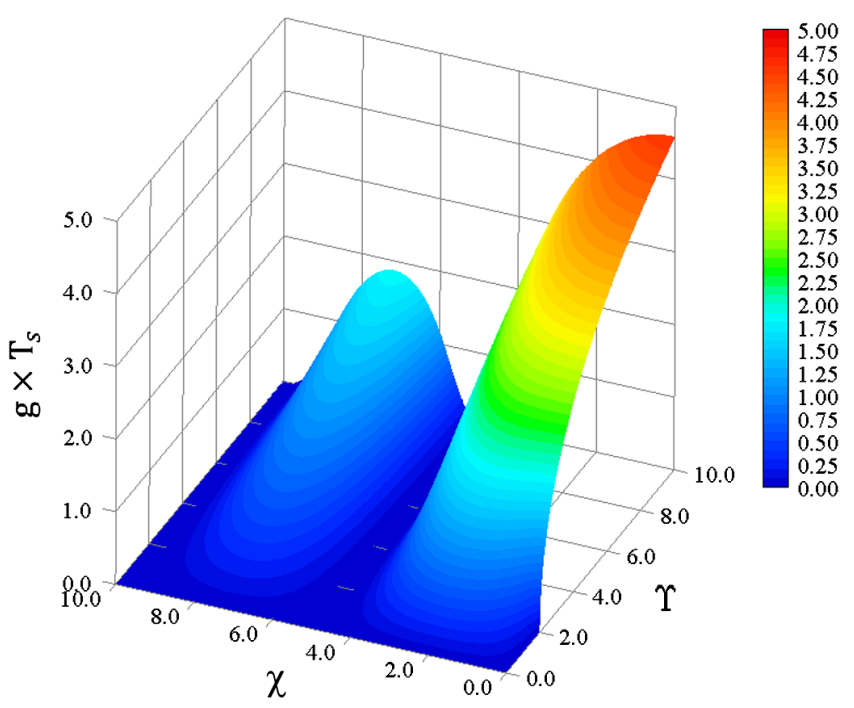

FIG. 7. Three-dimensional contour plot in the square well model for the growth factor $g \times T_{s}$ as a function of $\Upsilon$ and $\chi$ for positive $\chi$. 


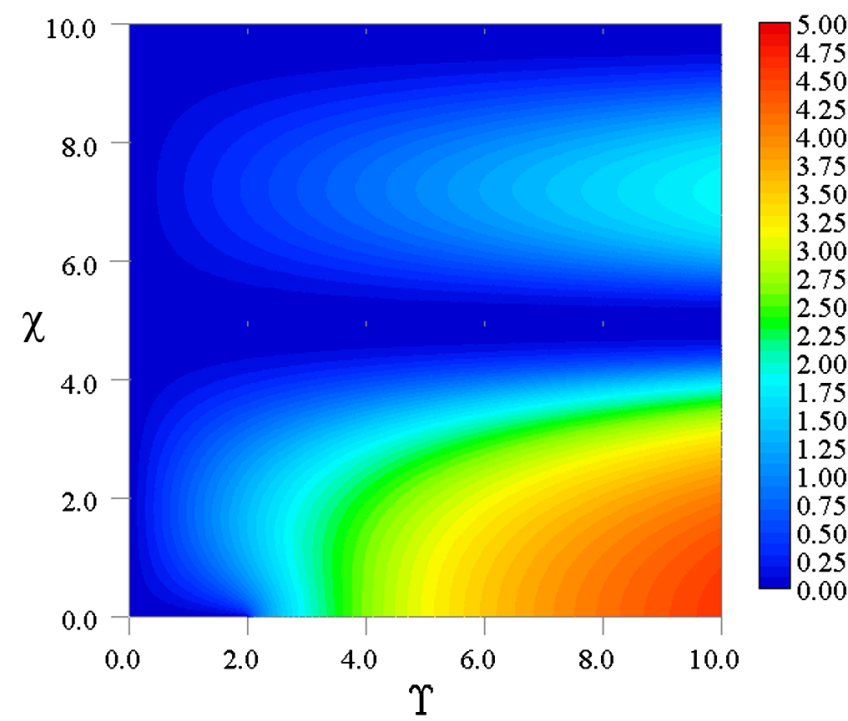

FIG. 8. Flat contour plot in the square well model for the growth factor $g \times T_{s}$ as a function of $\Upsilon$ and $\chi$ for positive $\chi$.

\section{FINDINGS WITH THE TWO PARTICLE MODEL}

As pointed out in Chao's book, the presented two particle model provides the symmetrical behavior of the growth rate for positive and negative $\chi$. In the Vlasov equation analyses and real machines, slightly positive and negative values for the chromaticity are preferred for stability of beams for operation above and below transition, respectively. This discrepancy arises since the \pm modes are treated equally in the two particle model, while they behave differently in real machines since the frequency of the pure dipole mode shifts always downward. Despite this drawback, the present two approaches provide the simple but powerful tools for further studies.

For small $\Upsilon$ and $\chi$, the growth factor can be approximated as [see Eq. (4.99) on page 201, in Ref. [2]]

$$
g \times T_{s}=\frac{2}{\pi} \Upsilon_{\chi}
$$

Figure 9 shows the growth factor $g \times T_{s}$ when $\Upsilon$ and $\chi$ are less than 0.5 (hereafter, we limit our discussions to the parabolic potential case only; similar discussions can be made for the square well potential case). It agrees with the formula (64) with very good accuracy.

From Fig. 9, it can be seen that the chromaticity excites head-tail instabilities even at very small wakefields, and their growth rates are proportional to the chromaticity. As pointed out in Chao' book, very roughly speaking, the real and the imaginary part of $\Gamma$ [they come from the functions $h(\chi)$ and $g(\chi)$, respectively] are responsible for the strong head-tail instability (or TMCI) and the head-tail instability, respectively. Since both functions are continuous functions, a clear threshold for the strong head-tail instability is hard

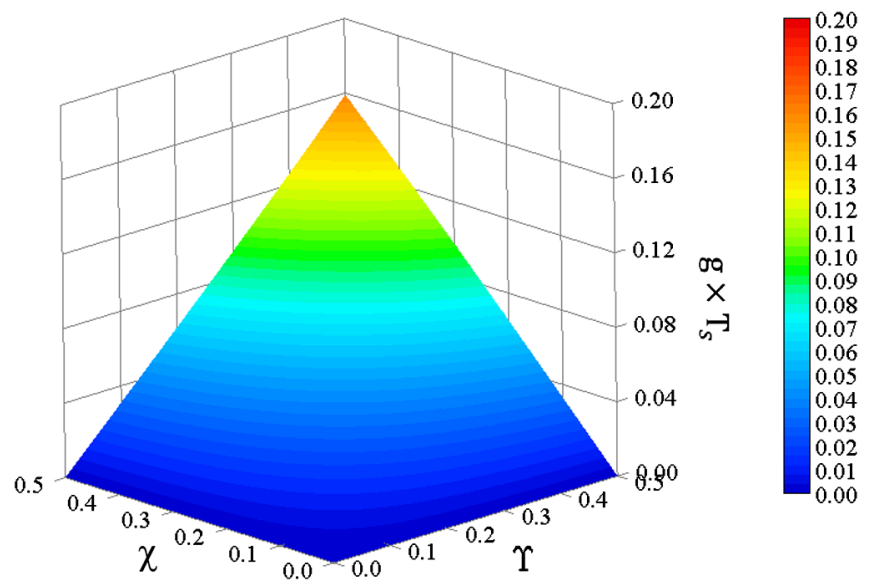

FIG. 9. The growth factor $g \times T_{s}$ as a function of $\Upsilon$ and $\chi$ when they are less than 0.5 .

to identify. However, we can make the following observations from Figs. 2 and 3: (i) The strong head-tail instability with a large growth rate appears mostly at $\chi$ below 4 . (ii) Around the lines along $\chi=4.33$ and 6.78, head-tail instability is strongly suppressed even at a large $\Upsilon$ (strong wakefields), say $\mathrm{r}=6$.

The values $\chi=4.33$ and 6.78 are roots of the function $g(\chi)$. To illustrate the second observation more clearly, we magnify the 3D contour plot (Fig. 2) up to $\Upsilon=6$ and plot it in Fig. 10 from a different observing angle. The threshold value $\mathrm{Y}$ for the strong head-tail instability at zero chromaticity is $\Upsilon=2$. The growth rates along the $\chi=4.33$ and 6.78 lines are very small even at $\Upsilon=6$. This observation indicates that it may be possible to mitigate both the strong head-tail instability and the head-tail instability simultaneously for rather strong wakefields, by carefully choosing the chromaticity along the $\chi=4.33$ and 6.78 lines. In the

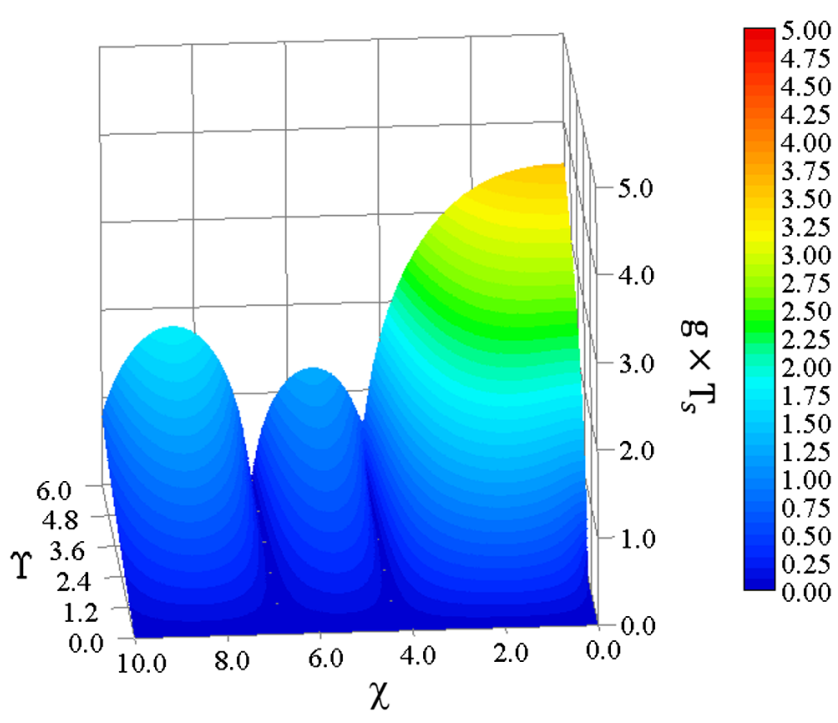

FIG. 10. Three-dimensional contour plot for the growth factor $g \times T_{s}$ as a function of $\Upsilon$ and $\chi$ from an angle different from Fig. 2 . 
next section, we will apply the Vlasov approach (with a Gaussian bunch distribution) to the present problem and produce contour plots for two wake potential models.

The present two particle model has only two modes. That may be enough to describe two lowest head-tail modes, the $m=0$ and the $m=1$ modes. However, it is not clear if it can accurately represent higher-order head-tail modes, since they profile more than two nodes in the oscillation envelops, while the two particles can make only one node at most. Roughly speaking, the mode spectrum [7] has peaks at $\chi \approx(m+1) \pi$ for $m \geq 1$. Thus, if $\chi$ exceeds $2 \pi$, higherorder head-tail modes start to be excited. In this sense, $\chi \approx 2 \pi$ may be an effective upper range of the present twoparticle analysis.

\section{VLASOV ANALYSIS}

In this section, we apply the Vlasov approach to the present problem to see how head-tail instabilities behave in a more elaborate model as a function of $\Upsilon$ (or the bunch current) and $\chi$. In this regard, we employ two wake potential models: the "constant" wake potential model and the broadband resonator model. For the direct comparison with the two particle model, we start with the constant wake potential model. We assume a Gaussian bunch distribution hereafter.

If we assume that the wake potential is constant forever behind the bunch, its impedance has a singularity at zero frequency [Eq. (6.215) on page 350 in Chao's book]:

$$
Z_{T}(\omega)=W_{0}\left[\frac{1}{\omega}-i \pi \delta(\omega)\right] .
$$

It diverges at zero frequency. However, the constant wake potential model does not really require that the wake potential needs to be constant forever. It should be constant only inside the bunch, since we are dealing with single bunch instability and particles within the bunch will not experience wakefields behind the bunch. Thus, any assumption for the wake potential behind the bunch should not change the instability behavior. It can be zero.

In this regard, we employ the trapezoidal model for the constant wake as illustrated in Fig. 11. It starts with zero (as required by the causality) and quickly reaches the constant value $W_{0}$ within the rise time of $z_{r} / c$. Then, after the distance $Z_{W}$, it comes back to zero again with the fall time of $z_{r} / c$. If we identify $Z_{W}$ as the total bunch length $2 \hat{z}$, and take the limit of $z_{r} \rightarrow 0$, the transverse impedance for the trapezoidal wake model becomes

$Z_{T}(\omega)=2 W_{0} \hat{z} / c\left[\frac{\sin ^{2} \omega \hat{z} / c}{\omega \hat{z} / c}-i \frac{\sin \omega \hat{z} / c \cdot \cos \omega \hat{z} / c}{\omega \hat{z} / c}\right]$.

We note that Eq. (66) is a standard approximation for the transverse impedance of a stripline beam position monitor of length $\hat{z}$.

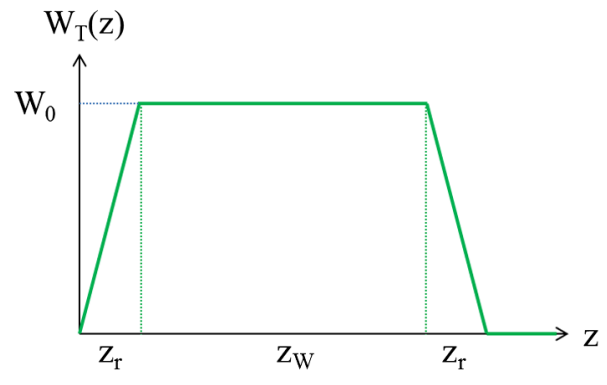

FIG. 11. The trapezoidal model for the constant wake approximation.

Figure 12 shows how this impedance behaves as a function of $\omega \hat{z} / c$ (the angular frequency times $\hat{z} / c$ ). The real part (the solid line) starts with zero, and after taking the peak, it gradually declines while oscillating. It stays always positive. On the other hand, the imaginary part (the dashed line) starts with negative value (inductance) and changes its sign to positive (capacitance). It then oscillates between positive and negative values while slowly declining. This behavior is similar to that of a broadband resonator impedance model (the direct comparison will be shown later in Fig. 20), except at high frequency: the trapezoidal wake model needs large high frequency impedance components to produce the fast rise and fall of the wake potential at the head and tail of the bunch.

The next step is to show that when the trapezoidal wake model is adopted in the Vlasov formulation, its solutions scale as a function of the three dimensionless parameters, $\chi, \Upsilon$, and the growth factor $g \times T_{s}$, as in the two particle model. A proof is given in Appendix D (for simplicity, we assumed the airbag bunch model in this proof; a similar proof can be made for a Gaussian bunch model).

The MOSES code [8,9], which is widely used for calculations of TMCI, assumes the resonator impedance model. We extended it to include the trapezoidal impedance model for the present study. The extended version is named

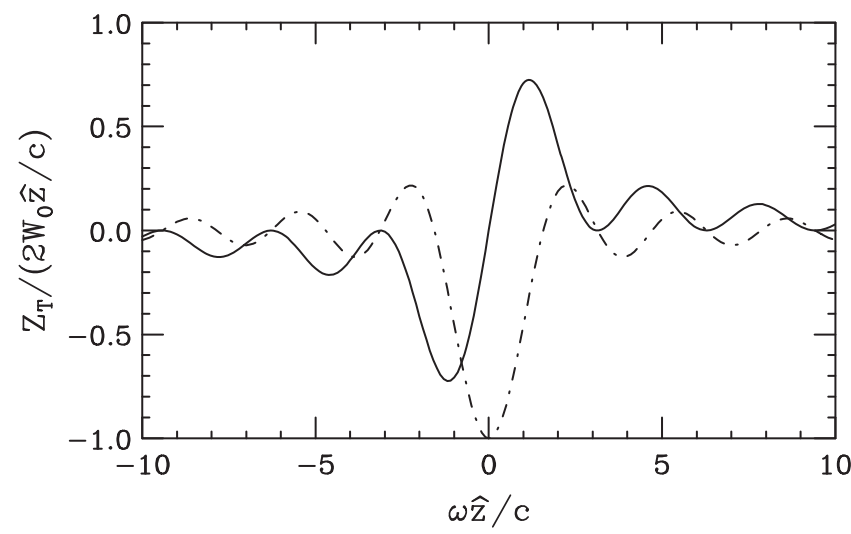

FIG. 12. The transverse impedance of the trapezoidal wake model. The real and the imaginary parts of the impedance are shown by the solid and the dashed lines, respectively. 
"MOSCOW" (MOSES for the COnstant Wake). We refer to this name hereafter.

We still have to find out what the reasonable definition of the bunch length $\hat{z}$ is in the Gaussian distribution, which has an infinitely long tail. The bunch length $\hat{z}$ determines the duration of the constant wake, $Z_{W}(=2 \hat{z})$, in the trapezoidal wake model. The parameter $Z_{W}(=2 \hat{z})$ should be long enough so that the wake potential behind it does not change the behavior of the instability. Thus, the minimum length of $Z_{W}(=2 \hat{z})$ that satisfies this condition can be found by gradually increasing $Z_{W}(=2 \hat{z})$ in the instability calculation with MOsCow and sizing up at which $Z_{W}(=2 \hat{z})$ the instability behavior saturates. For this end, let us introduce the parameter TFCT, the length of $\hat{z}$ in unit of the rms bunch length, $\sigma_{z}$. If TFCT is 3, the three sigma of the Gaussian distribution, which amounts to $99.73 \%$ of the Gaussian distribution, is covered by the flattop of the trapezoidal wake model. Thus, it is likely that TFCT $=3$ is already long enough. Figure 13 shows the growth factor $g \times T_{s}$ as a function of $\Upsilon$ for various TFCTs at zero chromaticity. Hereafter, the growth factor refers to that of the most unstable mode. It can be seen that the growth factors for $\mathrm{TFCT}=2$ (the black line) and TFCT $=2.2$ (the blue line) are almost identical, while TFCT $=1.8$ (the green line) slightly differs from them. The TFCT $=1$ line (the red line) is far different from all the other lines. Therefore, we can conclude that the minimum length of the bunch length $\hat{z}$ that satisfies the requirement of the trapezoidal wake mode is $2 \sigma_{z}$ of the Gaussian distribution (in other words, the length of $Z_{W}$ is $4 \sigma_{z}$ ).

This exercise also helps us to define $\chi$, the difference of the betatron phase advances between the head and the tail in a Gaussian distribution:

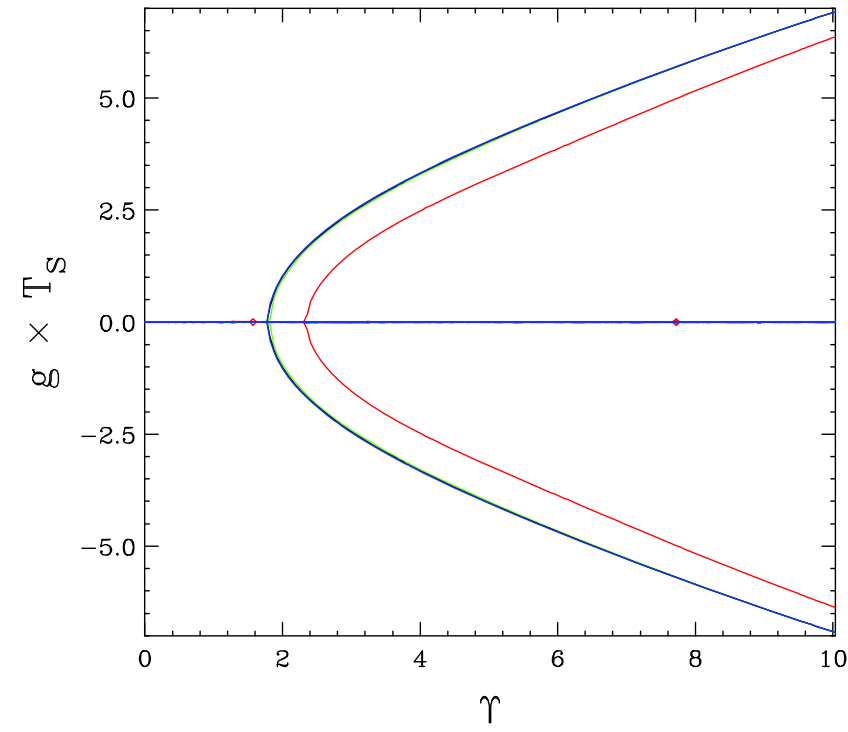

FIG. 13. The growth factor $g \times T_{s}$ (of the most unstable mode) as a function of $\Upsilon$ for various TFCTs (TFCT $=1$ by the red line, $\mathrm{TFCT}=1.8$ by the green lien, TFCT $=2$ by the black line, and $\mathrm{TFCT}=2.2$ by the blue line, respectively) at zero chromaticity.

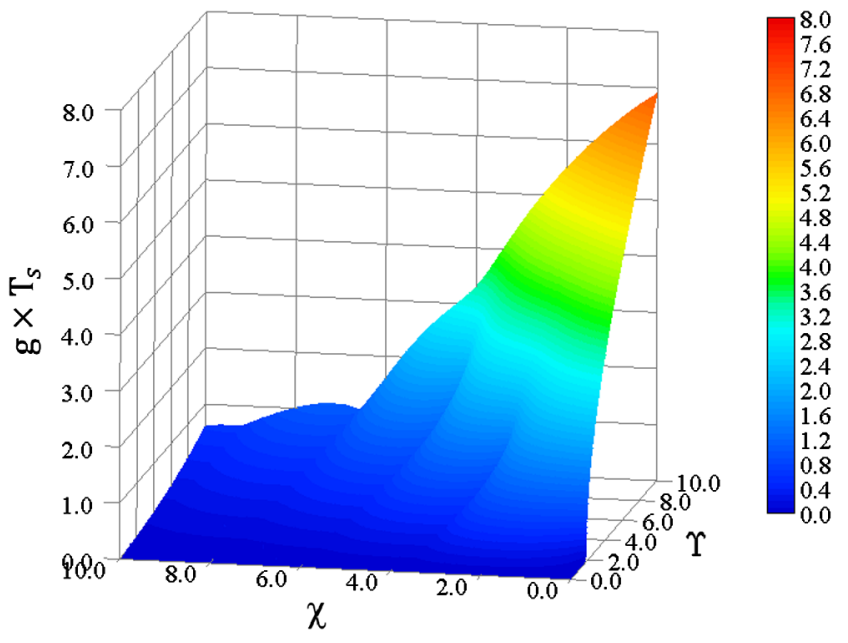

FIG. 14. The three-dimensional contour plot of the growth factor $g \times T_{s}$ (of the most unstable mode) as a function of $\Upsilon$ and $\chi$ at the rms bunch length of $2 \mathrm{~cm}$ in the trapezoidal wake model for the Vlasov analysis with the Gaussian bunch distribution.

$$
\chi=2 \frac{\xi \omega_{\beta}}{c \eta} \hat{z}=4 \frac{\xi \omega_{\beta}}{c \eta} \sigma_{z} .
$$

Now, we are ready to calculate contour plots for the growth factor $g \times T_{s}$ (of the most unstable mode) as a function of $\Upsilon$ and $\chi$, in the Vlasov analysis with a Gaussian bunch distribution. Figures 14 and 15 show the threedimensional and the flat contour plots of the growth factor $g \times T_{s}$ at the rms bunch length of $2 \mathrm{~cm}$, respectively [large numbers of azimuthal (from $m=-5$ to $m=5$ ) and radial (6) modes are used in the calculations to secure the saturation of results; we also specify the bunch length,

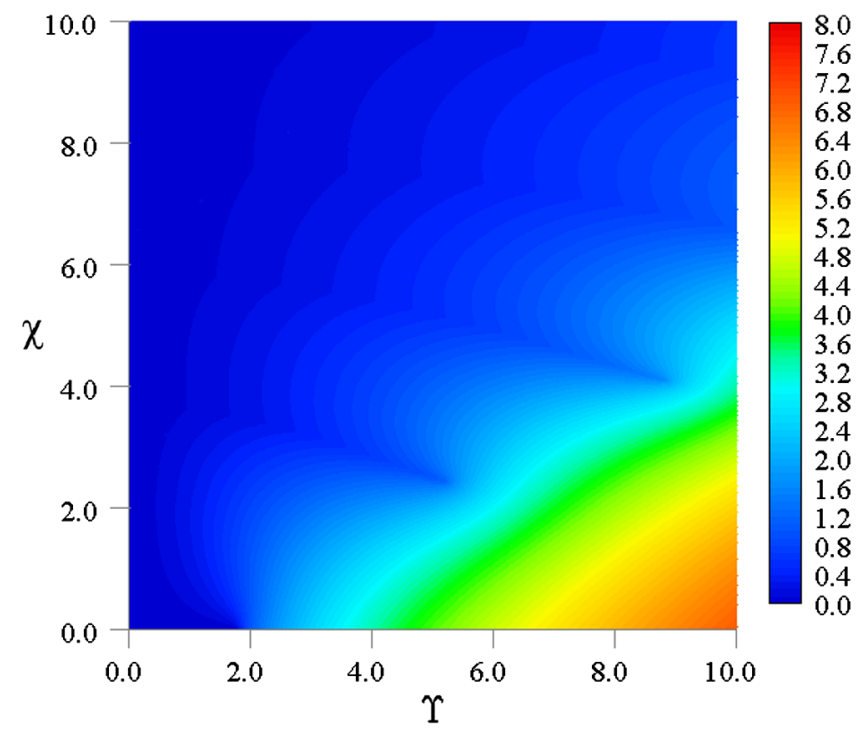

FIG. 15. The flat contour plot of the growth factor $g \times T_{s}$ (of the most unstable mode) as a function of $\Upsilon$ and $\chi$ at the rms bunch length of $2 \mathrm{~cm}$ in the trapezoidal wake model for the Vlasov analysis with the Gaussian bunch distribution. 
though the result has no bunch length effect as shown later in Fig. 18]. They should be compared with Figs. 2 and 3 for the two particle model, respectively. We observe that: (i) The unstable region at small $\chi$ becomes smaller and there is no other significantly unstable region at large $\chi$. (ii) The stable lines near $\chi=4.33$ and 6.78 still exit, but they are tilted downward in the direction of stronger wake. They no longer constitute large stable areas around them.

It seems that the two particle model exaggerates the effects of higher-order head-tail modes at large $\chi$ and the stability areas near the lines $\chi=4.33$ and 6.78. Despite the small differences in the details of the contour plots, their overall behaviors are quite similar between the two particle model and the Vlasov analysis.

Figure 16 shows a flat contour plot of the growth factor $g \times T_{s}$ as a function of $\Upsilon$ and $\chi$ for negative $\chi$. The growth factor is nonsymmetrical for positive and negative $\chi$ in the Vlasov analysis, as expected. It can be clearly seen that a negative chromaticity above the transition (namely, a negative $\chi$ ) works mostly to destabilize a beam, unless it is extremely large.

The contour plots should have no TFCT dependence as far as it is larger than 2, since the wake potential behind the bunch cannot alter the instability behavior. Figure 17 shows the three-dimensional contour plot for $\mathrm{TFCT}=4$. No significant difference can be seen from Fig. 14 for $\mathrm{TFCT}=2$. The contour plot for the trapezoidal wake model also should have no bunch length dependence, since the wake potential is constant within the bunch. Figure 18 shows the three-dimensional contour plot for the rms bunch length of $4 \mathrm{~cm}$. Again, no significant difference is visible from Fig. 14 for the rms bunch length of $2 \mathrm{~cm}$.

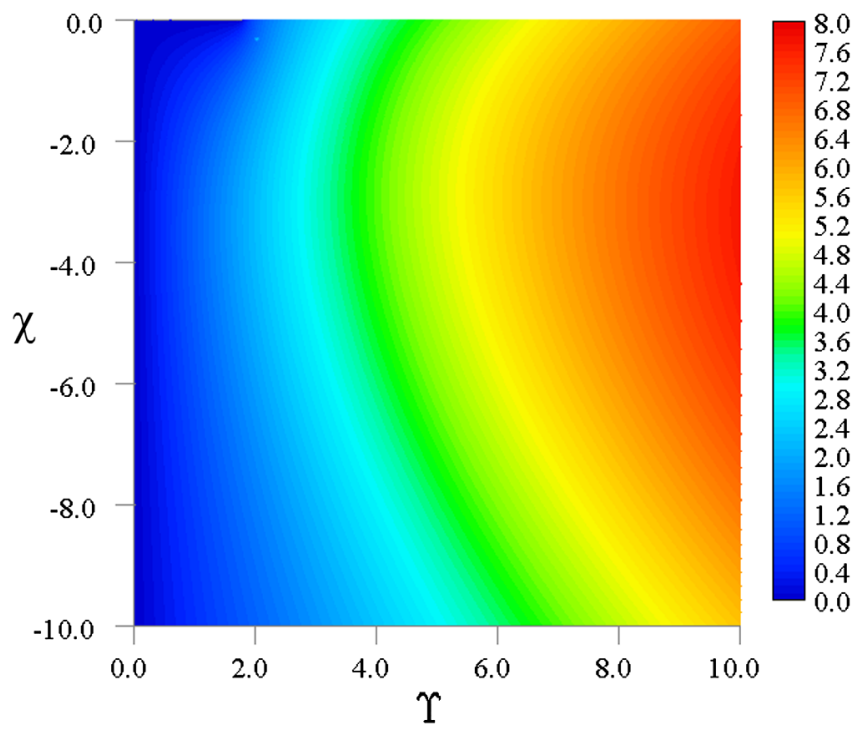

FIG. 16. The flat contour plot of the growth factor $g \times T_{s}$ (of the most unstable mode) as a function of $\Upsilon$ and negative $\chi$ at the rms bunch length of $2 \mathrm{~cm}$ in the trapezoidal wake model for the Vlasov analysis with the Gaussian bunch distribution.

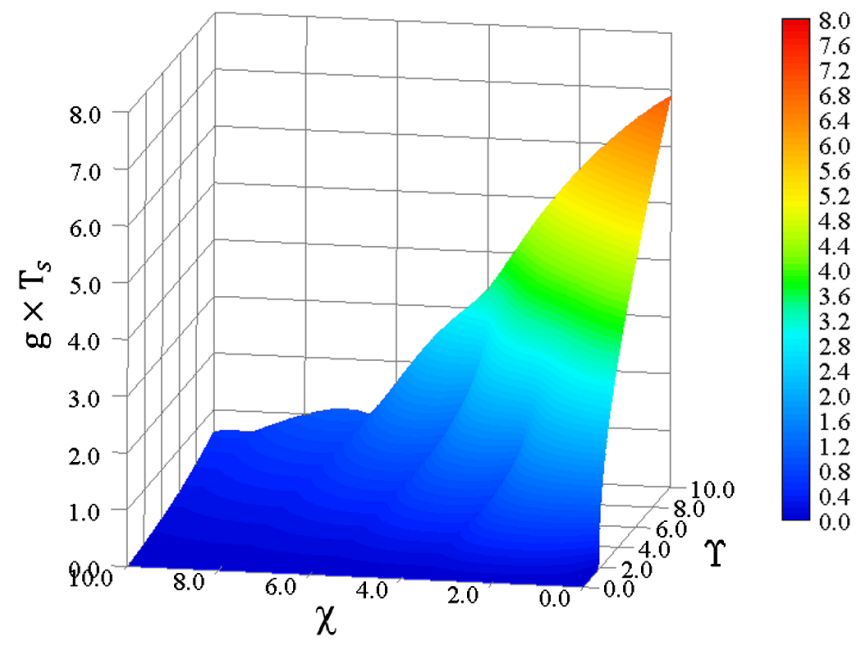

FIG. 17. The three-dimensional contour plot of the growth factor $g \times T_{s}$ as a function of $\Upsilon$ and $\chi$ for TFCT $=4$ at the rms bunch length of $2 \mathrm{~cm}$ in the trapezoidal wake model for the Vlasov analysis with the Gaussian bunch distribution.

Now, a question arises whether the trapezoidal wake model is a peculiar model since it assumes a constant wake potential within a bunch, which may sound oversimplified compared to more realistic wake potential models. To find out if this is the case, we also calculated contour plots for the LEP broadband resonator model: the resonator frequency $=2 \mathrm{GHz}$, the shunt impedance $=2 M \Omega / m$, and the $\mathrm{Q}$-value $=1$. This model has been frequently used for TMCI calculations at LEP [10,11]. The main LEP machine parameters used in this calculation are summarized in Table I. Figures 19 and 20 show the three-dimensional and the flat contour plots of the growth factor, respectively. They are strikingly similar to those of the trapezoidal wake model (Figs. 14 and 15), respectively.

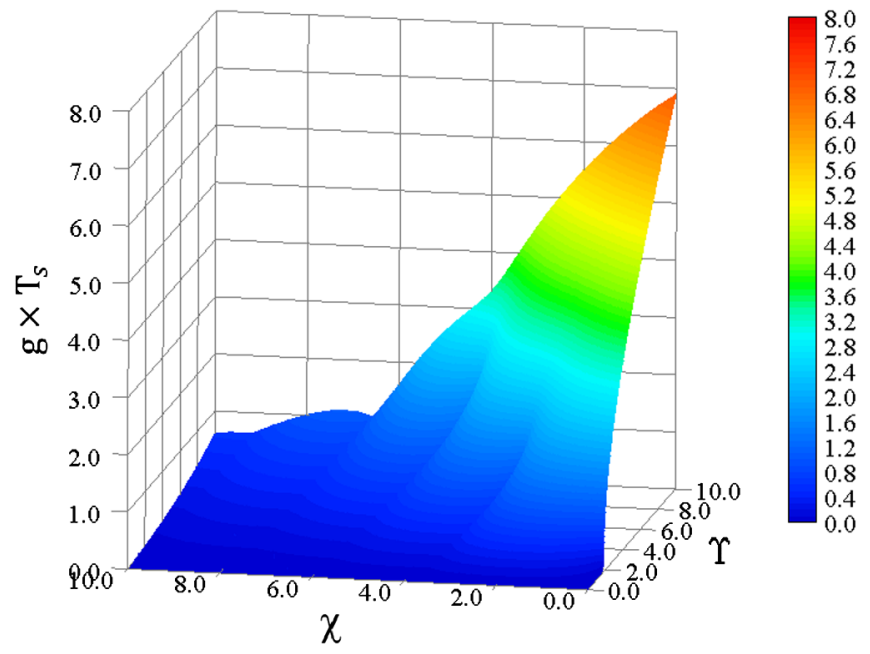

FIG. 18. The three-dimensional contour plot of the growth factor $g \times T_{s}$ as a function of $\Upsilon$ and $\chi$ at the rms bunch length of $4 \mathrm{~cm}$ in the trapezoidal wake model for the Vlasov analysis with the Gaussian bunch distribution. 
Table I. The main LEP machine parameters used in the contour plots.

\begin{tabular}{lc}
\hline \hline Energy & $20 \mathrm{GeV}$ \\
Revolution frequency & $11.246 \mathrm{kHz}$ \\
Momentum compaction factor & 0.0003867 \\
Beta function at the impedance location & $40.7 \mathrm{~m}$ \\
Synchrotron tune & 0.089931 \\
rms bunch length & $2 \mathrm{~cm}$ \\
\hline \hline
\end{tabular}

To find out the reason for this similarity, let us compare their impedances. For this end, we determine the parameter $W_{0}$ to have the same TMCI threshold bunch current $\left(I_{b}=0.705 \mathrm{~mA}\right)$ for the two models at zero chromaticity. Figure 21 shows their impedances. The black line is for the trapezoidal wake model $\left(\sigma_{z}=2 \mathrm{~cm}\right)$, and the red line is for the LEP broadband resonator impedance model, respectively. The real and the imaginary parts of the impedance are denoted by the solid and the dashed lines, respectively. They are quite similar at low frequency. The difference exists mostly at high frequency, where the trapezoidal wake model needs more high frequency impedance components to realize the fast rise and fall on the ends of the trapezoid wake. But, they contribute little to lower-order head-tail mode behaviors, since their mode spectrum is concentrated at low frequency. Figure 22 shows the wake potentials of the two models. Apart from the sharp edges at the rise and fall of the wake potential, they are overall comparable.

The striking resemblance of the contour plots between the trapezoidal wake model and the LEP resonator model indicates that the trapezoidal wake model is not an oversimplified model at all for short range wake effects. It has the reasonably behaved impedance and, most importantly, it substantially simplifies the time domain analysis.

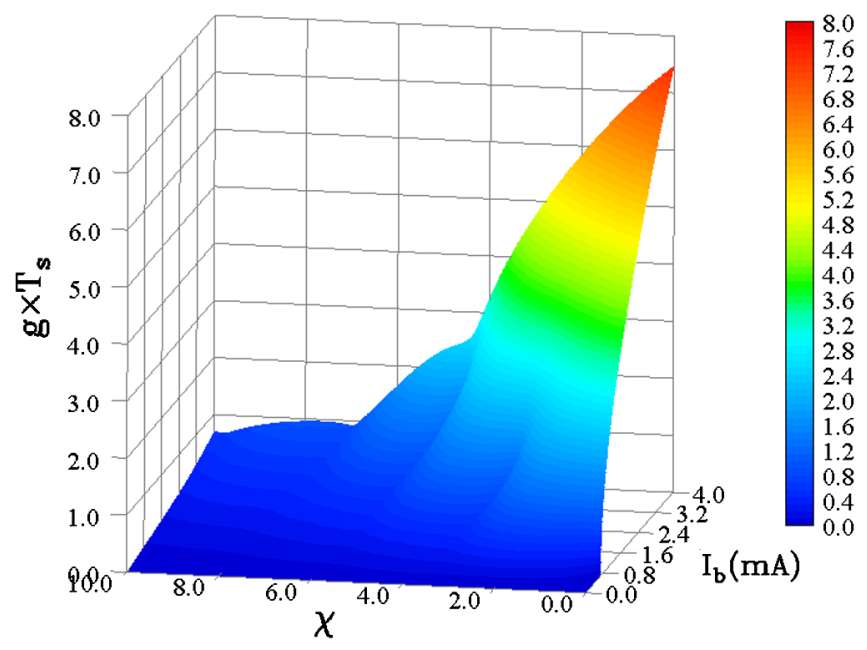

FIG. 19. The three-dimensional contour plot of the growth factor $g \times T_{s}$ as a function of $\Upsilon$ and $\chi$ at the rms bunch length of $2 \mathrm{~cm}$ in the LEP resonator model for the Vlasov analysis with the Gaussian bunch distribution.

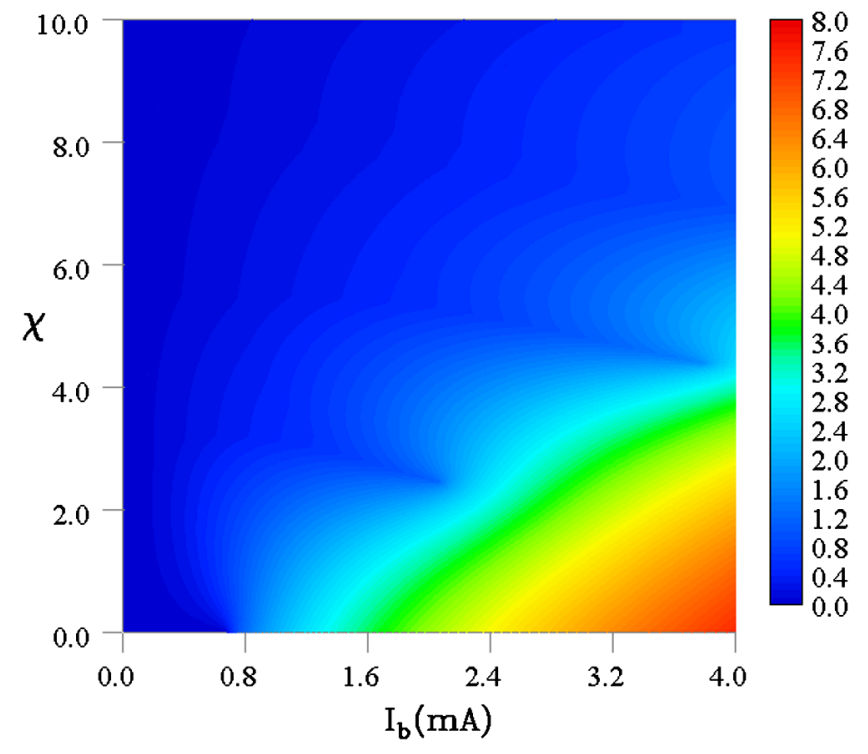

FIG. 20. The flat contour plot of the growth factor $g \times T_{s}$ as a function of $\Upsilon$ and $\chi$ at the rms bunch length of $2 \mathrm{~cm}$ in the LEP resonator model for the Vlasov analysis with the Gaussian bunch distribution.

\section{SIMULATIONS}

Lastly, we demonstrate that our findings with the Vlasov analysis are reinforced by simulation results. For this purpose, we have used the simulation code TRANFT $[12,13]$. In simulations, ten wakefield kicks are applied to a beam every turn. Figure 23 shows the growth factor $g \times T_{s}$ as a function of $\chi$ at $\Upsilon=2$, slightly above the threshold value $(=1.8)$ at zero chromaticity. The black line is the MOSCOW result, while the green line is the simulation result, for the trapezoidal wake model. They agree very well, in particular at large $\chi$. At small $\chi$, the simulations show slightly larger growth factor than the theory. This can

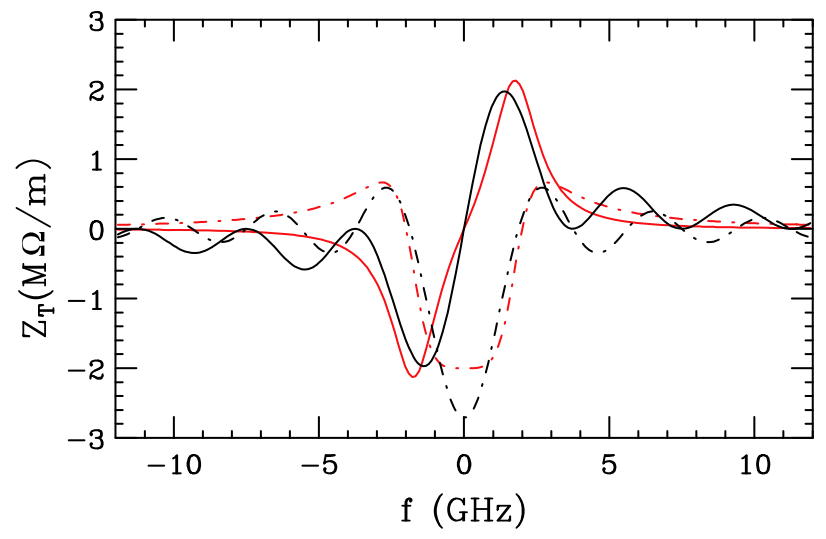

FIG. 21. The impedances of the two models. The black line is for the trapezoidal wake model $\left(\sigma_{z}=2 \mathrm{~cm}\right)$, and the red line is for the LEP broadband resonator impedance model, respectively. The real and the imaginary parts of the impedance are denoted by the solid and the dashed lines, respectively. 


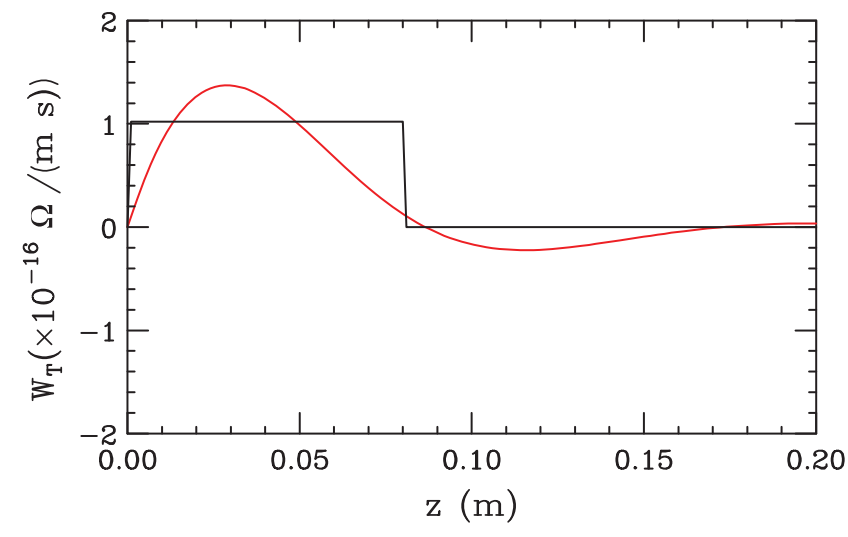

FIG. 22. The wake potentials of the two models. The black line is for the trapezoidal wake model $\left(\sigma_{z}=2 \mathrm{~cm}\right)$, and the red line is for the LEP broadband resonator impedance model, respectively.

be attributed to the fact that the instability growth rate is too fast at small $\chi$ (the growth factor $=1$ means that the amplitude of dipole oscillation grows by a factor of $e=$ 2.72 within one synchrotron oscillation period, which is only 11 turns). Thus, it is difficult to determine precise values of the growth factor at small $\chi$ in the simulations.

The red line in Fig. 23 is the MOSCOW result for the LEP broadband resonator model at the bunch length of $2 \mathrm{~cm}$ and at the bunch current of $0.78 \mathrm{~mA}$ that corresponds to $\Upsilon=2$. It is slightly larger than those of the constant wake models at large $\chi$, but its overall behavior is similar. The blue line is the simulation result for the LEP broadband resonator model. It agrees well the theoretical result (the red line) for the same model.

Now, let us look at the case of $\Upsilon=1.5$, slightly below the threshold value $(=1.8)$ at zero chromaticity. In this case, the growth factor $g \times T_{s}$ starts with zero and grows for a

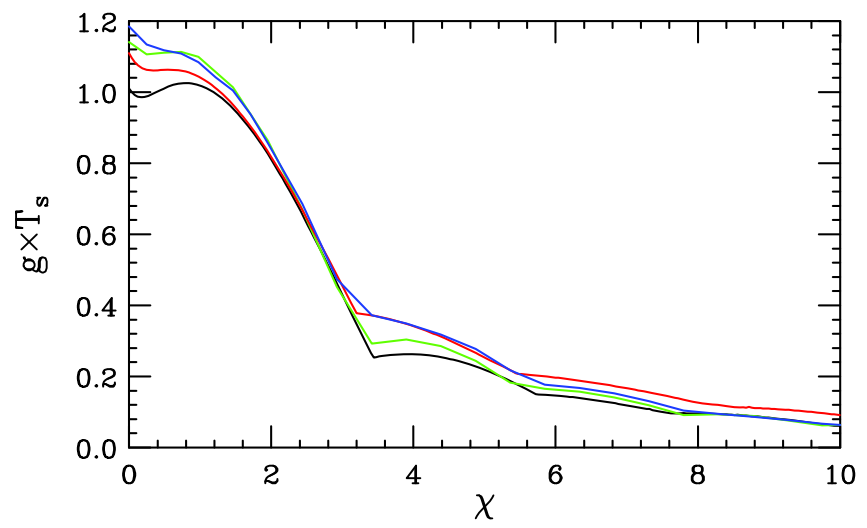

FIG. 23. The growth factor $g \times T_{s}$ as a function of $\chi$ at $\Upsilon=2$. The black line is the Moscow result, while the green line is the simulation result, for the trapezoidal wake model. The red line is the MOSCOW result for the LEP broadband resonator model at the bunch length of $2 \mathrm{~cm}$ and at the bunch current of $0.78 \mathrm{~mA}$ that corresponds to $\Upsilon=2$. The blue line is the simulation result for the LEP broadband resonator model. while as a function of $\chi$, since the chromaticity excites unstable head-tail modes. Figure 24 shows the growth factor $g \times T_{s}$ as a function of $\chi$. The black and the green lines are the MOscow and the simulation results for the trapezoidal wake model, respectively. They agree with each other very well. The red and blue lines are the Moscow result and the simulation results for the LEP broadband resonator model, respectively, at the bunch length of $2 \mathrm{~cm}$ and at the bunch current of $0.585 \mathrm{~mA}$ that corresponds to $\Upsilon=1.5$. They are also in good agreement. Despite the different wake potential models, their behaviors of the growth factor $g \times T_{s}$ as a function of $\chi$ are very comparable.

At last, let us compare the Vlasov results with those of the two particle model. Since the threshold value of $\Upsilon$ in the two particle model at zero chromaticity is 2 , not 1.8 as in the Vlasov approach, we need to slightly inflate the values of $\Upsilon$ in the two particle model, namely from 2 to 2.2 (corresponding to Fig. 23) and from 1.5 to 1.7 (corresponding to Fig. 24). Figure 25 shows the growth factor $g \times T_{s}$ as a function of $\chi$ according to the two particle model. The blue and the green lines are for the $\mathrm{Y}=2.2$ and $\mathrm{X}=1.7$, respectively. One can see that the two particle model provides larger growth factors over a wide range of $\chi$, except the large drops around $\chi=4.33$ and 6.78, which corresponds to the "stable areas" observed in Sec. VIII. The appearance of the growth factor "mountains" at large $\chi$ can be explained by the following reason. The two particles in the two particle model play many modes, even higher-order head-tail modes. At large $\chi$, higher-order head-tail modes dominate in the beam instabilities, and two particles overplay their contributions.

It may be interesting to point out that the dips around $\chi=3.5$ and 5.5 in Figs. 23 and 24 for the Vlasov analysis correspond to the stable "valleys" around $\chi=4.33$ and

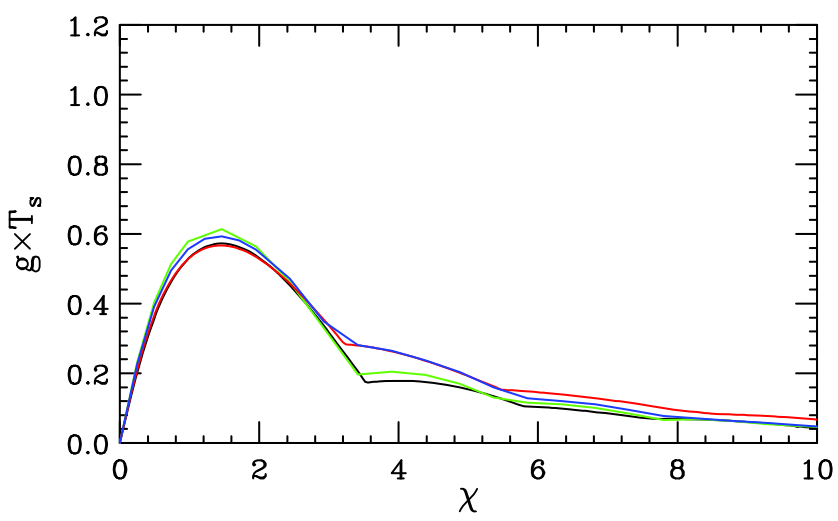

FIG. 24. The growth factor $g \times T_{s}$ as a function of $\chi$ at $\Upsilon=1.5$. The black line is the Moscow result, while the green line is the simulation result, for the trapezoidal wake model. The red line is the MOSCOW result for the LEP broadband resonator model at the bunch length of $2 \mathrm{~cm}$ and at the bunch current of $0.585 \mathrm{~mA}$ that corresponds to $\Upsilon=1.5$. The blue line is the simulation result for the LEP broadband resonator model. 


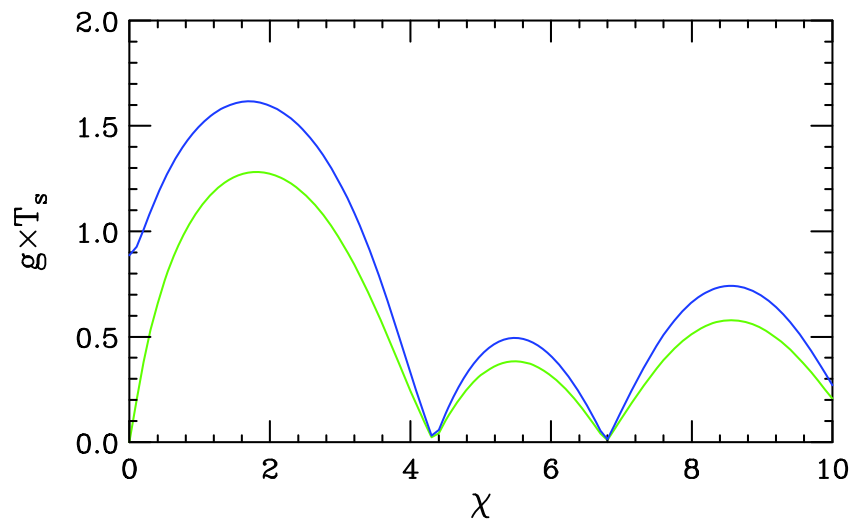

FIG. 25. The growth factor $g \times T_{s}$ as a function of $\chi$ according to the two particle model. The blue and the green lines are for the $\Upsilon=2.2$ and $\Upsilon=1.7$, respectively.

6.78 in Fig. 25, respectively. The mechanism of appearance of these dips at certain $\chi$ is worth studying.

\section{CONCLUSIONS}

Under the trapezoidal wake model, we succeeded to produce the universal contour plots of the growth factor $g \times T_{s}$ (of the most unstable mode) as a function of $\Upsilon$ and $\chi$ (Figs. 14 and 15) in the Vlasov analysis with a Gaussian bunch. They are similar to those in the two particle model (Figs. 2 and 3), though there are some differences in details. Figures 23 and 24 show the excellent agreements between the theory and the simulations. It also demonstrates that even at $\Upsilon=2$, slightly above the threshold value $(=1.8)$ at zero chromaticity, the beam is very unstable at small $\chi$ (the growth time is comparable to the synchrotron oscillation period), and thus it is unlikely that the unstable regions beyond $\Upsilon=2$ are actually reachable in real machines.

TMCI has been observed in rings (both electron and proton rings) only with relatively short bunches. In a short bunch, it needs a significantly larger chromaticity to produce a large $\chi$ (say, more than several). Figure 23 shows that the growth factor changes a little when $\chi$ stays within a few. It implies that the TMCI threshold can be hardly improved by increasing the chromaticity if it is within a reasonably attainable value (namely, $\chi \leq \mathrm{a}$ few). That may explain why the chromaticity has not been an effective tool to mitigate TMCI in many machines.

The striking resemblance of the contour plots between the trapezoidal model (Figs. 14 and 15) and the LEP broadband resonator model (Figs. 19 and 20) demonstrates that the trapezoidal wake model can give quantitatively reasonable predictions, while it can significantly simplify the time domain analysis by having the constant wake potential within a bunch. Figures 23 and 24 illustrate that this finding is supported by the simulation results. It may be safe to say that the universal contour plots with trapezoidal wake model (Figs. 14 and 15) show overall accurate pictures on how the beam instabilities behave as a function of $\Upsilon$ (or the bunch current) and $\chi$ (or the chromaticity). The trapezoidal wake model also has the well-behaved impedance as shown in Fig. 21. We believe that our findings on the trapezoidal wake model will help to advance the instability theories further by its simplicity and good impedance behavior.

\section{ACKNOWLEDGMENTS}

We would like to thank Olga Konstantinova for helping with the development of the Moscow code. This work was supported by U.S. Department of Energy 431 Contracts No. DE-SC0012704 and No. DEAC02-76SF00515.

\section{APPENDIX A: EQUATION OF MOTION FOR PARTICLE 2}

The derivative of $\hat{y}_{2}$ is

$$
\begin{aligned}
\hat{y}_{2}^{\prime} & =y_{2}^{\prime}+i\left(-2 \alpha_{2} y_{2}^{\prime}+\beta_{2} y_{2}^{\prime \prime}+\alpha_{2}^{\prime} y_{2}+\alpha_{2} y_{2}^{\prime}\right) \\
& =y_{2}^{\prime}+i\left(-\alpha_{2} y_{2}^{\prime}+\beta_{2} y_{2}^{\prime \prime}+\alpha_{2}^{\prime} y_{2}\right) .
\end{aligned}
$$

Here

$$
\alpha_{2}^{\prime}=\beta_{2}\left(K_{2}-\frac{1+\alpha_{2}^{2}}{\beta_{2}^{2}}\right)
$$

Thus,

$$
\begin{aligned}
\hat{y}_{2}^{\prime} & =y_{2}^{\prime}+i\left[-\alpha_{2} y_{2}^{\prime}+\beta_{2} y_{2}^{\prime \prime}+\beta_{2}\left(K_{2}-\frac{1+\alpha_{2}^{2}}{\beta_{2}^{2}}\right) y_{2}\right] \\
& =y_{2}^{\prime}\left(1-i \alpha_{2}\right)-i \frac{1+\alpha_{2}^{2}}{\beta_{2}} y_{2}+i \beta_{2} W y_{1},
\end{aligned}
$$

where we have used

$$
y_{2}^{\prime \prime}+K_{2} y_{2}=W y_{1}
$$

Since

$$
y_{2}^{\prime}=\frac{1}{i \beta_{2}}\left[\hat{y}_{2}-\left(1+i \alpha_{2}\right) y_{2}\right],
$$

we have

$$
\hat{y}_{2}^{\prime}=\frac{1}{i \beta_{2}} \hat{y}_{2}\left(1-i \alpha_{2}\right)+i \beta_{2} W y_{1} .
$$

Let us define

$$
\hat{y}_{2}(s)=a_{2}(s) \sqrt{\beta_{2}(s)} \exp \left[-i \int_{0}^{s} \frac{d s}{\beta_{2}(s)}\right] \text {. }
$$


Then,

$$
\hat{y}_{2}^{\prime}=a_{2}^{\prime} \sqrt{\beta_{2}} \exp \left[-i \int_{0}^{s} \frac{d s}{\beta_{2}}\right]+\frac{1}{i \beta_{2}} \hat{y}_{2}\left(1-i \alpha_{2}\right) .
$$

By comparing Eqs. (A6) and (A8), we obtain

$$
a_{2}^{\prime}(s) \sqrt{\beta_{2}} \exp \left[-i \int_{0}^{s} \frac{d s}{\beta_{2}}\right]=i \beta_{2} W y_{1}
$$

Here,

$$
\begin{aligned}
y_{1} & =a_{1} \sqrt{\beta_{1}} \cos \int_{0}^{s} \frac{d s}{\beta_{1}} \\
& =\frac{a_{1}}{2} \sqrt{\beta_{1}}\left[\exp \left(i \int_{0}^{s} \frac{d s}{\beta_{1}}\right)+\exp \left(-i \int_{0}^{s} \frac{d s}{\beta_{1}}\right)\right] .
\end{aligned}
$$

Thus,

$$
\begin{aligned}
a_{2}^{\prime}(s)= & i \sqrt{\beta_{1} \beta_{2}} \frac{W}{2} a_{1}\left[\exp \left(i \int_{0}^{s} \frac{d s}{\beta_{1}}+i \int_{0}^{s} \frac{d s}{\beta_{2}}\right)\right. \\
& \left.+\exp \left(-i \int_{0}^{s} \frac{d s}{\beta_{1}}+i \int_{0}^{s} \frac{d s}{\beta_{2}}\right)\right] .
\end{aligned}
$$

By performing the integral, we have

$$
\begin{aligned}
a_{2}(s)= & a_{2}(0)+i \frac{W}{2} a_{1}(0) \int_{0}^{s} \sqrt{\beta_{1} \beta_{2}}\left[\operatorname { e x p } \left(i \int_{0}^{s} \frac{d s}{\beta_{1}}\right.\right. \\
& \left.\left.+i \int_{0}^{s} \frac{d s}{\beta_{2}}\right)+\exp \left(-i \int_{0}^{s} \frac{d s}{\beta_{1}}+i \int_{0}^{s} \frac{d s}{\beta_{2}}\right)\right] d s .
\end{aligned}
$$

\section{APPENDIX B: DERIVATION OF EQ. (36)}

Let us put

$$
I(s) \stackrel{\text { def }}{=} \int_{0}^{s} \exp \left(i \chi \sin \frac{\omega_{s}}{c} s\right) d s=\frac{c}{\omega_{s}} \int_{0}^{z} \exp (i \chi \sin z) d z
$$

Using the formula

$$
\begin{aligned}
\exp (i \chi \sin z) & =\sum_{m=-\infty}^{\infty}(-1)^{m} J_{m}(\chi) e^{-i m z} \\
& =J_{0}(\chi)+\sum_{m \neq 0}\left[(-1)^{m} J_{m}(\chi) e^{-i m z}\right]
\end{aligned}
$$

we obtain

$$
I=\frac{c}{\omega_{s}}\left\{J_{0}(\chi) z+\sum_{m \neq 0}\left[(-1)^{m} J_{m}(\chi) \frac{1}{-i m}\left(e^{-i m z}-1\right)\right]\right\} .
$$

Here $J_{m}(\chi)$ is the Bessel function of the first kind. Since

$$
\begin{aligned}
& (-1)^{-m} J_{-m}(\chi) \frac{1}{i m}\left[e^{i m z}-1\right]+(-1)^{m} J_{m}(\chi) \frac{1}{-i m}\left[e^{-i m z}-1\right] \\
& =-(-1)^{m} \frac{2 i}{m} J_{m}(\chi) \begin{cases}(1-\cos m z) & \text { for } m=\text { odd }>0 \\
i \sin m z & \text { for } m=\text { even }>0,\end{cases}
\end{aligned}
$$

the function $I(s)$ can be written as

$$
\begin{aligned}
I(s)= & J_{0}(\chi) s-\frac{c}{\omega_{s}} \sum_{m=1}^{\infty}(-1)^{m} \frac{2 i}{m} J_{m}(\chi) \\
& \times \begin{cases}\left(1-\cos m \frac{\omega_{s}}{c} s\right) & \text { for } m=\text { odd } \\
i \sin m \frac{\omega_{s}}{c} s & \text { for } m=\text { even } .\end{cases}
\end{aligned}
$$

At $s=\frac{\pi c}{\omega_{s}}$, only the summation over odd integer survives and the function $I$ becomes

$$
I\left(\frac{\pi c}{\omega_{s}}\right)=\frac{\pi c}{\omega_{s}}\left[J_{0}(\chi)+i \sum_{\substack{m=1 \\ m=\text { odd }}}^{\infty} J_{m}(\chi) \frac{4}{m \pi}\right]
$$

By inserting this result into Eq. (35) and defining the dimensionless wakefield parameter $\Upsilon$ as Eq. (37), we obtain Eq. (36).

\section{APPENDIX C: SQUARE WELL MODEL}

For the two potential models to be comparable, the average value of the difference of the betatron phases between the two particles has to be equal in the two models. For this end, let us calculate the difference of the betatron phases between the two particles over a quarter of the synchrotron oscillation period. In the square potential model, it is 


$$
\int_{0}^{\frac{T_{s}}{4}} \frac{\omega_{\beta} \xi}{c \eta} \hat{z}_{s} \omega_{s}\left(\frac{s}{c}\right) d s /\left(\frac{T_{s}}{4}\right)=\frac{\omega_{\beta} \xi}{c \eta} \frac{\pi}{4} \hat{z}_{s},
$$

while in the parabolic potential model, it is

$$
\int_{0}^{\frac{T_{s}}{4}} \frac{\omega_{\beta} \xi}{c \eta} \hat{z} \sin \left(\omega_{s} \frac{s}{c}\right) d s /\left(\frac{T_{s}}{4}\right)=\frac{\omega_{\beta} \xi}{c \eta} \frac{2}{\pi} \hat{z} .
$$

Thus,

$$
\hat{z}_{s}=\frac{8}{\pi^{2}} \hat{z}
$$

Let us assume

$$
y_{m}(s)=\hat{y}_{m}(s) \exp \left(-i \omega_{\beta} \frac{s}{c}\right), \quad m=1 \quad \text { or } 2 .
$$

If we insert Eq. (C4) into Eqs. (55) and (56) and neglect the $\hat{y}_{m}^{\prime \prime}(s)$ term by assuming that $\hat{y}_{m}(s)$ changes slowly in time, we have for the first half of the synchrotron oscillation period $0<s / c<T_{s} / 2$, or $0<s<\pi c / \omega_{s}$,

$$
\hat{y}_{1}^{\prime}-i \frac{\omega_{\beta}}{c} \varepsilon_{s} c(s) \hat{y}_{1}=0,
$$

and

$$
\hat{y}_{2}^{\prime}+i \frac{\omega_{\beta}}{c} \varepsilon_{s} c(s) \hat{y}_{2}=\frac{i W c}{2 \omega_{\beta}} \hat{y}_{1},
$$

where

$$
\varepsilon_{s}=\frac{\xi \omega_{s} \hat{z}_{s}}{c \eta},
$$

and

$$
c(s)= \begin{cases}1, & 0<s<\pi c / 2 \omega_{s} \\ -1, & \pi c / 2 \omega_{s}<s<\pi c / \omega_{s} .\end{cases}
$$

The solution of Eq. (C5) is

$$
\hat{y}_{1}(s)=\hat{y}_{1}(0) \exp \left(i \frac{\omega_{\beta}}{c} \varepsilon_{s} d(s)\right),
$$

where

$$
d(s)= \begin{cases}s, & 0<s<\pi c / 2 \omega_{s} \\ \frac{\pi c}{\omega_{s}}-s, & \pi c / 2 \omega_{s}<s<\pi c / \omega_{s} .\end{cases}
$$

Multiplying Eq. (C6) by $\exp \left(i \frac{\omega_{\beta}}{c} \varepsilon_{s} d(s)\right)$, we get

$$
\begin{aligned}
\frac{d}{d s} & {\left[\hat{y}_{2}(s) \exp \left(i \frac{\omega_{\beta}}{c} \varepsilon_{s} d(s)\right)\right] } \\
& =\frac{i W c}{2 \omega_{\beta}} \hat{y}_{1}(0) \exp \left(2 i \frac{\omega_{\beta}}{c} \varepsilon_{s} d(s)\right) .
\end{aligned}
$$

Therefore, the transformation of $\hat{y}_{m}(s)$ over a half synchrotron oscillation period is

$$
\hat{y}_{1}\left(\pi c / \omega_{s}\right)=\hat{y}_{1}(0),
$$

and

$$
\begin{aligned}
\hat{y}_{2}\left(\pi c / \omega_{s}\right)= & \hat{y}_{2}(0)+\hat{y}_{1}(0) \frac{i W c}{2 \omega_{\beta}} \\
& \times \int_{0}^{\pi c / \omega_{s}} \exp \left(2 i \frac{\omega_{\beta}}{c} \varepsilon_{s} d(s)\right) d s .
\end{aligned}
$$

Notice that

$$
\begin{aligned}
& \int_{0}^{\pi c / \omega_{s}} \exp \left(2 i \frac{\omega_{\beta}}{c} \varepsilon_{s} d(s)\right) d s \\
& =2 \int_{0}^{\pi c /\left(2 \omega_{s}\right)} \exp \left(i \frac{\omega_{\beta}}{c} \varepsilon_{s} s\right) d s=\frac{\pi c}{2 \omega_{s}} \exp (i \Delta) \frac{\sin \Delta}{\Delta},
\end{aligned}
$$

where

$$
\Delta=\frac{\omega_{\beta}}{c} \varepsilon_{s} \frac{\pi c}{4 \omega_{s}} .
$$

Using the definition of $\varepsilon_{s}$ [Eq. (C7)] and the relation (C3), the parameter $\Delta$ can be written as

$$
\Delta=\frac{2}{\pi} \cdot 2 \frac{\xi \omega_{\beta}}{c \eta} \hat{z}=\frac{2}{\pi} \chi
$$

By inserting Eqs. (C14) and (C16) to Eq. (C13) and using the definition of $\Upsilon$ [Eq. (37)], Eq. (C13) can be written as

$$
\hat{y}_{2}\left(\pi c / \omega_{s}\right)=\hat{y}_{2}(0)+\hat{y}_{1}(0) \cdot i \Upsilon f_{s}(\chi),
$$

where 


$$
f_{s}(\chi)=\exp \left(i \frac{2 \chi}{\pi}\right) \cdot \frac{\sin \frac{2 \chi}{\pi}}{\frac{2 \chi}{\pi}} .
$$

The transfer matrix from $s=0$ to $s=\pi c / \omega_{s}$ is thus given by

$$
\left[\begin{array}{l}
\hat{y}_{1} \\
\hat{y}_{2}
\end{array}\right]_{s=\pi c / \omega_{s}}=\left[\begin{array}{ll}
1 & 0 \\
i \Gamma & 1
\end{array}\right]\left[\begin{array}{l}
\hat{y}_{1} \\
\hat{y}_{2}
\end{array}\right]_{s=0},
$$

where $\Gamma=\Upsilon \cdot f_{s}(\chi)$.

\section{APPENDIX D: PROOF THAT VLASOV SOLUTIONS SCALE WITH $\chi, \Upsilon$, and $g \times T_{s}$ IN THE TRAPEZOIDAL WAKE MODEL}

In this section, we prove that the Vlasov solutions with the trapezoidal wake model scale with $\chi, \Upsilon$, and $g \times T_{s}$. For simplicity, we assume the airbag model for the bunch distribution. The proof can be made for other bunch distribution models.

We start with the matrix formula for the coherent frequencies of head-tail modes [Eqs. (6.221) and (6.222) on page 353 in Chao's book]:

$$
\operatorname{det}\left(\frac{\Omega-\omega_{\beta}}{\omega_{s}}-M\right)=0
$$

where the matrix elements of $M$ are given by

$$
\begin{aligned}
M_{l l^{\prime}}= & l \delta_{l l^{\prime}}-i \frac{N r_{0} c^{2}}{4 \pi \gamma C \omega_{\beta} \omega_{s}} i^{l-l^{\prime}} \int_{-\infty}^{\infty} d \omega Z_{T}(\omega) J_{l} \\
& \times\left(\frac{\omega \hat{z}}{c}-\frac{\chi}{2}\right) J_{l^{\prime}}\left(\frac{\omega \hat{z}}{c}-\frac{\chi}{2}\right) .
\end{aligned}
$$

Here, we have used that $C=c T_{0}$ and added the chromaticity effect term $\chi / 2$. Notice that our definition of $\chi$ is a factor of 2 different from Chao's book where $\chi$ is defined by

$$
\chi=\frac{\xi \omega_{\beta} \hat{z}}{c \eta} .
$$

The transverse impedance of the trapezoidal wake model is

$$
Z_{T}(\omega)=2 W_{0} \hat{z} / c\left[\frac{\sin ^{2} \omega \hat{z} / c}{\omega \hat{z} / c}-i \frac{\sin \omega \hat{z} / c \cdot \cos \omega \hat{z} / c}{\omega \hat{z} / c}\right] .
$$

If we insert Eq. (D4) into Eq. (D2) and change the integral from $\omega$ to $y=\omega \hat{z} / c$, we have

$$
\begin{aligned}
M_{l l^{\prime}}= & l \delta_{l l^{\prime}}-i \frac{N r_{0} c^{2} W_{0}}{2 \pi \gamma C \omega_{\beta} \omega_{s}} i^{l-l^{\prime}} \int_{-\infty}^{\infty} d y \\
& \times\left[\frac{\sin ^{2} y}{y}-i \frac{\sin y \cdot \cos y}{y}\right] J_{l}\left(y-\frac{\chi}{2}\right) J_{l^{\prime}}\left(y-\frac{\chi}{2}\right) .
\end{aligned}
$$

This equation can be written as

$$
M_{l l^{\prime}}=l \delta_{l l^{\prime}}-i \frac{2 \Upsilon}{\pi^{2}} i^{l-l^{\prime}} F_{l l^{\prime}}(\chi),
$$

where

$$
\begin{aligned}
F_{l l^{\prime}}(\chi)= & \int_{-\infty}^{\infty} d y\left[\frac{\sin ^{2} y}{y}-i \frac{\sin y \cdot \cos y}{y}\right] \\
& \times J_{l}\left(y-\frac{\chi}{2}\right) J_{l^{\prime}}\left(y-\frac{\chi}{2}\right),
\end{aligned}
$$

and we have used the definition of $\Upsilon$ [Eq. (37)].

Therefore the eigensolutions $\frac{\Omega-\omega_{\beta}}{\omega_{s}}$ are determined only by $\Upsilon$ and $\chi$. The growth factor is given by

$$
g \times T_{s}=2 \pi \frac{\operatorname{Im}(\Omega)}{\omega_{s}} .
$$

Thus, the growth factor is also a function only of $\Upsilon$ and $\chi$.

[1] Y.H. Chin and K. Satoh, Transverse mode-coupling in a bunched beam, IEEE Trans. Nucl. Sci. 30, 2566 (1983); Transverse mode coupling in a bunched beam, Nucl. Instrum. Methods Phys. Res. 207, 309 (1983).

[2] A. Chao, Physics of Collective Beam Instabilities in High Energy Accelerators (John Wiley \& Sons, Inc., New York, 1993).

[3] R. Kohaupt, DESY Report No. M-80/19, 1980.

[4] R. Talman, The influence of finite synchrotron oscillation frequency on the transverse head-tail effect, Nucl. Instrum. Methods Phys. Res. 193, 423 (1982).

[5] C. Lee and S. Peggs, Report No. RHIC/AP/158, 1998.

[6] E. Wilson and E. J. N. Wilson, An Introduction to Particle Accelerators (Oxford University Press, Oxford, 2001), p. 32.

[7] J. Gareyte and F. Sacherer, in Proceedings of the 9th International Conference on High Energy Accelerators, Stanford (Stanford Univ., Stanford, 1974), p. 34.

[8] Y. H. Chin, Report No. CERN/LEP-TH/88-05, 1988.

[9] Y.H. Chin, MOSES home page, http://abci.kek.jp/moses .htm.

[10] D. Brandt et al., in Proceedings of EPAC90, Nice, France (Centre Antoine Lacassagne, Nice, 1990), p. 240. 
[11] B. Zotter, in Proceedings of the 3rd Workshop on LEP Performance, Chamonix, France (CERN, Geneva, 1993), p. 89.

[12] M. Blaskiewicz, A multipurpose coherent instability simulation code, in Proceedings of the 22nd Particle Accelerator Conference, PAC-2007, Albuquerque, NM
(IEEE, New York, 2007), THPAS090, p. 3690, http:// accelconf.web.cern.ch/AccelConf/p07/PAPERS/THPAS090 .PDF.

[13] M. Blaskiewicz, https://www.bnl.gov/isd/documents/ 32667.pdf. 